\title{
Relative Dynamics of Charged Spacecraft in a Magnetic Field applied to Formation Flight
}

\author{
Yu Cheng ${ }^{\mathrm{a}, \mathrm{b}, *}$, Gerard Gómez ${ }^{\mathrm{c}}$, Josep J. Masdemont ${ }^{\mathrm{d}}$, Dong Qiao ${ }^{\mathrm{a}, \mathrm{b}}$, Yijun \\ $\operatorname{Lian}^{\mathrm{e}}$ \\ ${ }^{a}$ School of Aerospace Engineering, Beijing Institute of Technology, 5 South Zhongguancun \\ Street, Beijing, 100081, China \\ ${ }^{b}$ Key Laboratory of Dynamics and Control of Flight Vehicle, Ministry of Education, Beijing, \\ 100081, China \\ ${ }^{c}$ IEEC \& Department de Matemàtiques i Informàtica, Universitat de Barcelona, Gran Via \\ 585, 08007 Barcelona, Spain \\ dIEEC \& Department de Matemàtiques, Universitat Politècnica de Catalunya, Diagonal \\ 647, 08028 Barcelona, Spain \\ e School of Aeronautical and Astronautical Science, National University of Defense \\ Technolog, Yanwachi 47, Changsha, 410073, China
}

\begin{abstract}
We consider several charged spacecraft configurations around a leader spacecraft provided with an artificial magnetic field. In all configurations the nominal orbits are chosen to be about the relative equilibrium points of the reference model. Using a linear quadratic regulator controller, we find that controllability is possible in most of the cases, and it is strongly related to the stability of the equilibrium point which, in turn, depends on the orbit of the leader and the rotation rate of its magnetic dipole.

The dynamical model considered shows a great potential for future formation flying applications since both, the establishment and maintenance of the configurations, can be managed by merely adjusting the charge on the follower spacecraft. No additional control variables are needed.
\end{abstract}

Keywords: Formation flight, Lorentz force, Formation keeping, Relative equilibrium point

\footnotetext{
* Corresponding author

Email address: chengyu8907@gmail.com (Yu Cheng)
} 


\section{Introduction}

The Lorentz force experienced by a charged spacecraft moving within a magnetic field, due to either a natural planetary magnetic filed or an artificial one induced by space-borne superconducting wires, provides a novel and propellant less way for the orbital control of charged spacecrafts. Compared with the traditional chemical propulsion, Lorentz force offers more advantages in fuel saving and, consequently, more capability for scientific payload, making it a promising technology for future space missions, including formation flying.

The use of Lorentz forces for spacecraft propulsion was first proposed in [16], inspired by the fact that the ballistic trajectory of a charged missile is affected by the geomagnetic field [8], and also because the Lorentz force perturbation on the dust grains, induced by the planetary magnetic fields, contributes to the formation of Jupiter and Saturn rings [26]. Without providing a detailed exploration, in [16] the authors proposed various applications for a "Lorentz spacecraft", including stable satellite formations using equilibrium locations, inclination change control for non-equatorial orbits, and Jovian capture using the Jupiter's strong magnetic field for spacecraft braking from a parabolic orbit.

A number of researches have been triggered by the Lorentz force actuated missions. For instance, in [29] the authors analytically determine new polar and equatorial Earth-synchronous orbits using the geomagnetic field, they also analyse the effect of the Lorentz force on equatorial gravity-assist manoeuvres [31]. They conclude that not only the Lorentz force can extend the influence of a planetary flyby, but it can also provide more flexibility in flyby timing through temporary capture. Anot

In [5] the authors found an analytical control law for the charge of the spacecraft to accomplish propellant less station-keeping at the Saturn-Enceladus L1 Lagrange point, which is a suitable location for constant observation of Enceladus. Later, they developed an analytical expression of the evolution of the orbital elements during Jupiter capture using the Lorentz force as the only perturbation, as well as the actuation force [7]. The Lorentz force assisted Jupiter 
capture problem has been explored both numerically in [1], and analytically in [31, 7], considering only the equatorial capture case. Other Lorentz force applications include inclination change [20], rendezvous [21] and planetary escape $[16,6]$, just to mention a few.

Formation flying using Lorentz forces has also been investigated by several authors. This idea was first introduced in [17], that explored the possibility, using this force, of doing reconfiguration manoeuvres for low Earth orbit proximity (25m) formations. The J2-invariant formations, using the Lorentz force induced by the geomagnetic field, was considered in [22], while in [39] the dynamics and control of Lorentz force spacecraft for missions of hovering, formation flying, and rendezvous was studied.

In [34 the authors found that the linearised system of a LEO formation is not controllable when the Lorentz force is the only source of propulsion; they developed a step wise charge control law for formation reconfiguration transfers. Due to the uncontrollability, in [32] Sobiesiak and Damaren investigated the formation keeping problem, taking into account the J2 perturbation, using hybrid propulsion that combines the Lorentz force with impulsive thrust; they extended their study to optimal formation reconfigurations [33]. While the aforementioned researches mainly focused on the orbital control using the Lorentz force induced by the geomagnetic field, in [35, 36] the authors found that the lack of controllability also exists in the attitude control of spacecraft moving in the Earth's magnetic field using magnetic torque. To address this problem, Yang [37] proposed an efficient algorithm to solve the periodic discrete-time Riccati equations, taking full advantage of the fact that the Earth's magnetic field can be approximated as periodic function for the spacecraft.

However, the previous researches have mainly considered natural planetary magnetic fields, such as the geomagnetic one and Jupiter's magnetic field. We recall that the Lorentz force is perpendicular to the instantaneous velocity of the charged spacecraft with respect to the magnetic filed and the magnetic field itself. This inherent property puts limitations on the direction of the force it can provide, and also restricts the motion of the spacecraft to certain regions within 
the planetary magnetic field. The idea of artificial Electro Magnetic Formation Flight (EMFF) was introduced in [12] to avoid the aforementioned domain restrictions. With this idea, each satellite can produce its own electromagnetic field, the interacting forces and torques can be used to control the size, orientation, and rotation of the configuration. In this area, Umair et al. 38 addressed the control strategy for electromagnetic formation maintenance and reconfiguration on near-Earth orbits, taking into account the effect of the geomagnetic field. In [13] the capability of EMFF for close proximity inspection missions was explored, and concluded that EMFF combined with reaction wheels can provide the forces and torques required for translation and attitude control needed for the formation maintenance. In [19] the feasibility of electromagnetic formation flight was tested in a microgravity environment for the first time, showing that axial attraction force can be generated between two electromagnets, however the main purpose was the hardware check and no control algorithm was considered.

Unlike EMFF, that uses the interaction between different electromagnetic fields as actuator, Peng [24] proposed a new formation flying scenario that uses as actuator the Lorentz force on a charged spacecraft induced by an artificial magnetic field deployed on the leader spacecraft of the formation. In their model, the leader can produce an artificial magnetic dipole by three concentric hightemperature super-conducting coils (HTSC) [24]; the three orthogonal coils are such that any orientation of the dipole can be achieved by tuning the current in the three HTSC. However, in the above reference, only a radially pointed magnetic dipole was considered, and for this configuration the study was focused in the equilibrium points and their stability, as well as in the periodic orbits emanating in the centre manifold of the equilibrium point. Later, in [25], a normally directed magnetic dipole was considered, in this case, the relative dynamics of the follower spacecraft was studied in order to construct bounded periodic orbits around the leader moving in the same orbital plane of the leader. In [3, 4] Cheng et al. extended the above study to three orientations of the dipole: normal, radial and tangential, and performed an extensive dynamical analysis, including the symmetries of the system, the equilibrium points and 
their stability, and the periodic and the quasi-periodic orbits emanating from these elliptic equilibria.

Concerning the technical issues for the accomplishment of Lorentz force actuated missions, the two main challenges are the spacecraft surface charging [15], and the thermal control system of the HTSC (see [4, 13, 2]). According to [27], with the current technology, the charge-to-mass ratio of the spacecraft is feasible in the range $10^{-6}$ to $10^{-3} \mathrm{C} / \mathrm{kg}$, while [16] states that the value $10^{-2} \mathrm{C} / \mathrm{kg}$ will be feasible in a near future.

Most of the existing literature on Lorentz force formation flying uses the Lorentz force as a control force or a mean of propulsion, this is, the Lorentz force is used to perform the transfer manoeuvres to reach the orbits solution of the HCW differential equations of relative motion, see, for instance, [34, 32, 33]. Several researchers have computed the relative equilibrium points with respect to the leader spacecraft in presence of the Lorentz force ([17, 34]), as well as some periodic orbits constructed by continuous charge variation [34]. Few studies (to the authors' best knowledge) have considered the station-keeping for those motions, one exception is [34], in which the considered equilibria are uncontrollable, thus the formation flying is not achievable using only the Lorentz force.

In a paper already mentioned [4], Cheng et al. analysed, using Dynamical Systems methods, the relative dynamics of a constantly charged follower spacecraft moving in the artificial magnetic dipole produced by the leader. Using these tools, all the equilibrium points, and several families of periodic orbits and quasi-periodic invariant tori were accurately determined, together with their stability properties. In the current paper we explore the application of those results to formation flying. Selecting suitable nominal locations around the equilibrium points, several formation flying configurations are designed and analysed. A linear feedback control law is applied for the formation keeping using only the Lorentz force. We note that the technical limitations already mentioned are not the concerns of this paper, and we assume that the chargeto-mass ratio, as well as the magnetic field required for close-proximity formation 
keeping, can be achieved.

The paper is organized as follows: the dynamical model is briefly introduced in Section 2, including the differential equations of motion for the three orientations of the dipole considered. Several configurations about the equilibrium points computed in [4] (consisting of a single or multiple spacecrafts, with either the same or different charges), are analysed in Section 3, including the effects of initial errors in their location. In Section 4, we consider the controllability of the linearised system around the equilibrium points, and we propose a linear quadratic regulator (LQR) feedback control law for the formation keeping, where the charge of the spacecraft is the only control variable.

The capability of self-maintenance of the formation is also examined for different stability types of the reference equilibria and different values of the initial displacements. Some discussions on the potential of the proposed formation flying model are also included, as well as some suggestions for future work. The conclusions are given in the last section of the paper. More possible formation flying configurations using as reference trajectories some periodic orbits computed in [4], will be discussed in a forthcoming paper.

We emphasize that in this paper, the artificial magnetic field is used to maintain the formation configuration, and the effect of the Earth's magnetic field on the charged follower is neglected, under the assumption that the orbit of the leader is supposed to be high enough (for instance, geostationary orbit).

\section{Reference model and equations of motion}

We consider a relative motion scenario where an electrostatically charged spacecraft (follower) moves about a leader spacecraft that produces a rotating artificial magnetic dipole (see [4] for details). We assume that the dipole is generated by three concentric and orthogonal superconducting wires, such that the orientation of the dipole can be in any direction by adjusting the current in the wires. Moreover, the follower has a negligible mass compared to the one of the leader and, thus, no influence is caused by the follower on the leader. Therefore, 
the follower is subject to the gravitational force of the Earth together with the Lorentz force induced by the dipole of the leader, while only the gravitational force of the Earth acts on the leader.

The reference system considered is the usual local-vertical-local-horizontal (LVLH) one (see Fig. 1): the origin is located at the leader, the $x$-axis $\left(\boldsymbol{e}_{r}\right)$ is along the leader's position vector along its circular orbit, the $z$-axis $\left(\boldsymbol{e}_{n}\right)$ is perpendicular to the orbital plane, and the $y$-axis $\left(\boldsymbol{e}_{t}\right)$ completes a right-hand coordinate frame.

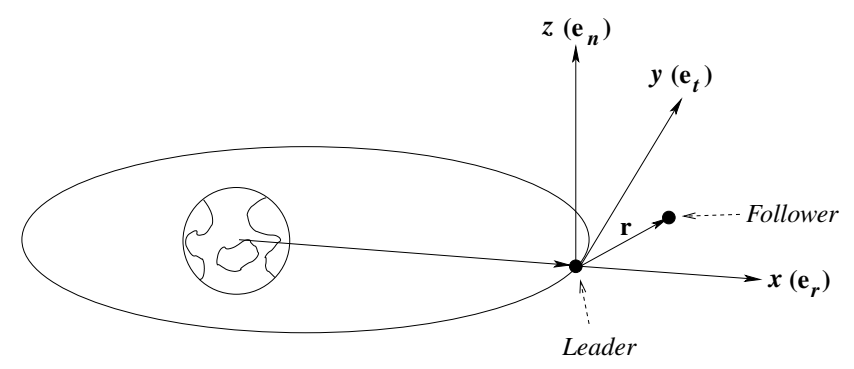

Figure 1: The Local-Vertical-Local-Horizontal (LVLH) reference system[4].

The equations of relative motion of the follower can be derived from the well-known Hill-Clohessy-Wiltshire (HCW) equations, adding an additional acceleration due to the Lorentz force acting on the follower. So the HCW equations become:

$$
\begin{aligned}
\ddot{x}-2 n \dot{y}-3 n^{2} x & =f_{x}, \\
\ddot{y}+2 n \dot{x} & =f_{y}, \\
\ddot{z}+n^{2} z & =f_{z},
\end{aligned}
$$

where $n$ is the mean motion of the leader's orbit around the Earth, and the Lorentz force $\boldsymbol{f}_{L}=\left(f_{x}, f_{y}, f_{z}\right)^{\top}$ is given by:

$$
\boldsymbol{f}_{L}=\frac{q}{m} \boldsymbol{v}_{r} \times \boldsymbol{B}
$$

where $\boldsymbol{v}_{r}$ is the charged follower's velocity relative to the leader's rotating magnetic dipole, $\boldsymbol{B}$ is the leader's rotating magnetic dipole, and $q / m$ is the charge- 
to-mass ratio, which is a key parameter to determine the magnitude of the Lorentz force.

According to the orientation of the dipole producing the magnetic field, we consider three cases:

- normal: the axis of the dipole $\boldsymbol{B}$ is parallel to $\boldsymbol{e}_{n}$,

- radial: the axis of the dipole $\boldsymbol{B}$ is parallel to the radius vector $\boldsymbol{e}_{r}$, and

- tangential: the axis of the dipole $\boldsymbol{B}$ is parallel to $\boldsymbol{e}_{t}$.

To simplify the equations of motion, we introduce the following units [4]: the dimensionless time unit $\tau^{\star}$, and the distance unit $a^{\star}$ are such that:

$$
\tau^{\star}=n t, \quad a^{\star}=\left|\frac{B_{0}}{n} \frac{q}{m} \frac{1}{\beta}\right|^{1 / 3},
$$

where $B_{0}$ is the magnetic moment of the dipole, and the parameter $\beta=n / \omega_{c}$ is the ratio between the mean motion $n$ of the leader in its circular orbit about the Earth and the angular velocity of the dipole, that is denoted by $\omega_{c}$.

Let $\tau=t / \tau^{\star}$ be the adimensional time, the time derivative with respect to $\tau$ is denoted by a prime $\left({ }^{\prime}=d / d \tau\right)$. Using the above units and notation, and defining the new position vector as $\left(X=x / a^{\star}, Y=y / a^{\star}, Z=z / a^{\star}\right)$, we can write the normalized equations of motion (see [4] for the details) as:

$$
\begin{aligned}
X^{\prime \prime}-2 Y^{\prime}-3 X & =f_{X}, \\
Y^{\prime \prime}+2 X^{\prime} & =f_{Y}, \\
Z^{\prime \prime}+Z & =f_{Z},
\end{aligned}
$$

where the right-hand side terms are the components of normalized Lorentz force, that can be expressed as:

$$
f_{X}=\frac{\sigma}{R^{5}} g_{x}^{*}, \quad f_{Y}=\frac{\sigma}{R^{5}} g_{y}^{*}, \quad f_{Z}=\frac{\sigma}{R^{5}} g_{z}^{*},
$$

where the components of the force-field $\left(g_{x}^{*}, g_{y}^{*}, g_{z}^{*}\right)$ are: $\left(g_{x}^{N}, g_{y}^{N}, g_{z}^{N}\right)$ in the normal case, in which the dipole axis $\boldsymbol{B}$ is parallel to $\boldsymbol{e}_{n} ;\left(g_{x}^{R}, g_{y}^{R}, g_{z}^{R}\right)$ in the radial case, in which the dipole axis $\boldsymbol{B}$ is parallel to the radius vector $\boldsymbol{e}_{r}$, and 
$\left(g_{x}^{T}, g_{y}^{T}, g_{z}^{T}\right)$ in the tangential case, in which the dipole axis $\boldsymbol{B}$ is parallel to $\boldsymbol{e}_{t}$. The expressions of $\left(g_{x}^{*}, g_{y}^{*}, g_{z}^{*}\right)$ for the three cases are given in Eqs. (5).

$$
\begin{aligned}
& g_{x}^{N}=-\beta\left(X^{2}+Y^{2}-2 Z^{2}\right) Y^{\prime}-3 \beta Y Z Z^{\prime}+X\left(X^{2}+Y^{2}-2 Z^{2}\right), \\
& g_{y}^{N}=\beta\left(X^{2}+Y^{2}-2 Z^{2}\right) X^{\prime}+3 \beta X Z Z^{\prime}+Y\left(X^{2}+Y^{2}-2 Z^{2}\right), \\
& g_{z}^{N}=3 Z\left[\beta Y X^{\prime}-\beta X Y^{\prime}+\left(X^{2}+Y^{2}\right)\right], \\
& g_{x}^{R}=3 X\left[\beta Z Y^{\prime}-\beta Y Z^{\prime}+\left(Y^{2}+Z^{2}\right)\right], \\
& g_{y}^{R}=-\beta\left(Y^{2}+Z^{2}-2 X^{2}\right) Z^{\prime}-3 \beta X Z X^{\prime}+Y\left(Y^{2}+Z^{2}-2 X^{2}\right), \\
& g_{z}^{R}=-\beta\left(Y^{2}+Z^{2}-2 X^{2}\right) Y^{\prime}+3 \beta X Y X^{\prime}+Z\left(Y^{2}+Z^{2}-2 X^{2}\right), \\
& g_{x}^{T}=\beta\left(X^{2}+Z^{2}-2 Y^{2}\right) Z^{\prime}+3 \beta Y Z Y^{\prime}+X\left(X^{2}+Z^{2}-2 Y^{2}\right), \\
& g_{y}^{T}=3 Y\left[\beta X Z^{\prime}-\beta Z X^{\prime}+\left(X^{2}+Z^{2}\right)\right], \\
& g_{z}^{T}=-\beta\left(X^{2}+Z^{2}-2 Y^{2}\right) X^{\prime}-3 \beta X Y Y^{\prime}+Z\left(X^{2}+Z^{2}-2 Y^{2}\right) .
\end{aligned}
$$

In the above equations $\sigma$ is the sign of the charge of the follower, and $R=$ $\sqrt{X^{2}+Y^{2}+Z^{2}}$.

Recall that the artificial magnetic field is generated by self-rotating HTSC coils, and, according to [13], the value of the magnetic moment $B_{0}$ is:

$$
B_{0}=\frac{\mu_{0}}{4} n_{c} i_{c} R_{c}^{2}
$$

where $\mu_{0}=4 \pi \times 10^{-7} \mathrm{~N} / \mathrm{A}^{2}$ is the vacuum permeability, $n_{c}$ is the number of loops in the coil, $i_{c}$ is the current in the coil, and $R_{c}$ is the coil radius.

Note that once the size $\left(n_{c}, R_{c}\right)$ of the HTSC is fixed, the strength of the magnetic filed is determined by the value of current $i_{c}$ flown in it, which is mainly limited by the working temperature and the critical current density of the material of the HTSC.

For the computations that follow, we assume that the leader spacecraft moves on a geostationary orbit with a radius of $42,164 \mathrm{~km}$, thus the mean motion is $n=7.2940 \times 10^{-5} \mathrm{rad} / \mathrm{s}$. Using the current launch ability, the state-of-art technology for HTSC wires [18] could provide a space-based magnetic field with the magnetic dipole momentum up to 17.1 Tesla.m ${ }^{3}$. As for the charge-tomass ratio, $q / m$, the available values that the existing charging technologies 
can achieve are $10^{-6} \sim 10^{-3}(\mathrm{C} / \mathrm{kg})$ [27], while a maximum of $0.03 \mathrm{C} / \mathrm{kg}$ seems feasible with the best materials [16]. Table 1 lists the values of the unit length for different values of $\beta$ and $q / m$.

Table 1: Values of the length unit $a^{\star}$ (in meters) for different values of $\beta$ and $q / m$.

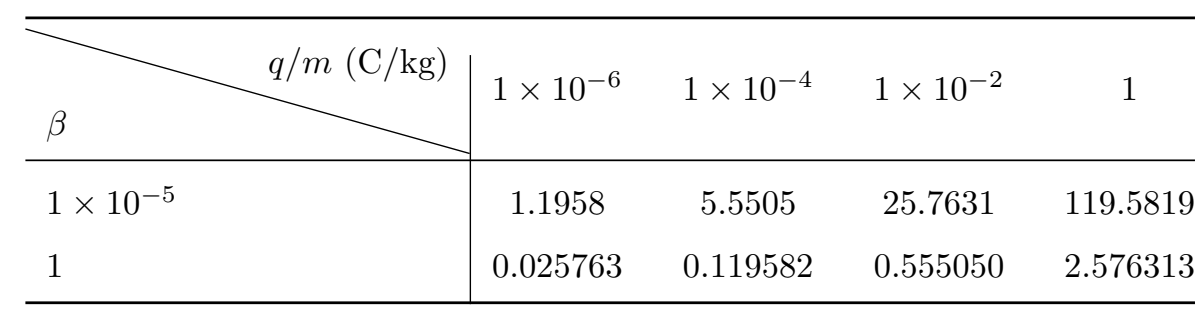

Taking into account that the Debye length for the GEO is of the order of $10 \mathrm{~m}$, in order to neglect the Coulomb force between charged spacecrafts, we will assume $q / m>10^{-2} \mathrm{C} / \mathrm{kg}$, and also that the distance between charged deputies will be larger than the Debye length.

\section{Formation flying configurations using equilibria}

In this section we define several formation flying configurations for a set of charged spacecrafts moving in the vicinity of one leader spacecraft provided with a magnetic field. We assume the the follower satellites are identical, both in mass and charge, and that the strength of the magnetic field is such that the distance unit $a^{\star}$ is of the order of several tenths of meters. In this way, the separation between two equilibrium points can be larger than the Debye length $(10 \mathrm{~m})$, and the Coulomb forces between two deputies can be neglected.

In the LVLH reference frame, the equilibria of the equations of motion (3) are stationary solutions relative to the leader. Many formation flight configurations can be defined using them as nominal locations for the spacecraft; furthermore, using different values for the charge $q$ of the deputies enlarges the number of possible configurations. In the normal and tangential case, there are 10 different equilibrium points, while the radial case system (3) has an infinite number of them. Fig. 2 shows their location in the normal, radial, and tangential case. 
The coordinates $(X, Y, Z)$ of the equilibrium points of these three systems of differential equations, are given in Table 2 as a function of the sign $\sigma$ of the charge $q$.

Table 2: Location of the equilibrium points in the normal $(\mathrm{N})$, radial $(\mathrm{R})$, and tangential case (T) as a function of the sign $\sigma$ of the charge ( see [4] for detail).

\begin{tabular}{ccccc} 
Label & $\sigma$ & $X$ & $Y$ & $Z$ \\
\hline $1_{N}$ & +1 & 0 & $\pm \sqrt{2} Z$ & $\pm\left(\frac{2}{3 \sqrt{3}}\right)^{1 / 3}$ \\
$2_{N}$ & +1 & $\pm\left(\frac{1}{12 \sqrt{6}}\right)^{1 / 3}$ & 0 & $\pm \sqrt{5} X$ \\
$3_{N}$ & -1 & $\pm\left(\frac{1}{3}\right)^{1 / 3}$ & 0 & 0 \\
\hline $1_{R}$ & +1 & 0 & 0 & \pm 1 \\
$2_{R}$ & -1 & $\pm\left(\frac{2}{9 \sqrt{3}}\right)^{1 / 3}$ & $\pm \sqrt{2} X$ & 0 \\
$3_{R}$ & -1 & $\pm\left(\frac{1}{4 \sqrt{2}}\right)^{1 / 3}$ & 0 & $\pm X$ \\
\hline $1_{T}$ & +1 & 0 & 0 & \pm 1 \\
$2_{T}$ & -1 & $\pm\left(\frac{1}{3}\right)^{1 / 3}$ & 0 & 0 \\
$3_{T}$ & \pm 1 & 0 & $\neq 0$ & 0 \\
\hline
\end{tabular}
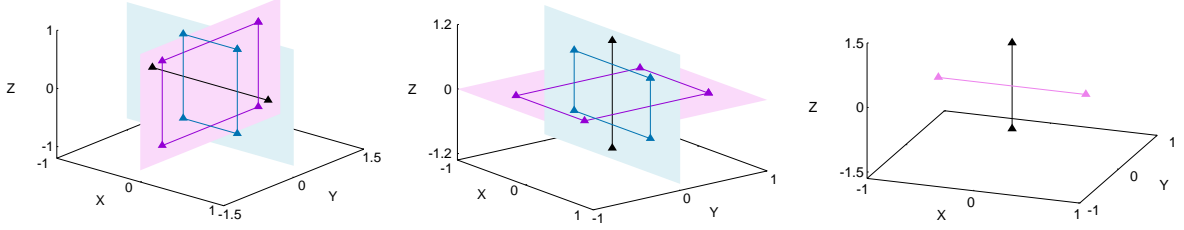

Figure 2: Some configurations, with the nominal locations of the spacecraft at the equilibria, in the normal case (left), the radial case (middle), and the tangential case (right). In each configuration, the followers (marked with small triangles) are located at the equilibria, while the segments link the adjacent satellites of a formation.

Recall that the distance unit is determined by the values of $q / m$ and $\beta$, and we have assumed that both $B_{0}$ and $\beta$ are fixed once we design the HTSC. In this way we can construct multi-satellite configurations using spacecraft with different charges $q$. For instance, using one of the rectangle configurations shown in Fig. 2, we can build a configuration with $3 \times 4=12$ spacecraft composed of 
3 similar rectangles, with a size ratio $m^{1 / 3}: n^{1 / 3}: p^{1 / 3}$, when the charges of the spacecraft of each rectangle are $q_{1}, q_{2}$, and $q_{3}$, with $q_{1}=m q^{\star}, q_{2}=n q^{\star}$, $q_{3}=p q^{\star}$.

Another option is to consider a string configuration, with several spacecraft of different charges along the same line,that goes from the origin (the leader) to one equilibrium point. Fig. 3 shows these two types of configurations using the $1_{N}$ equilibrium points, that are located at the four vertex of the magenta rectangle in the $Y-Z$ plane, as shown in Fig.2. In this way, we have four spacecrafts with $m=1$ (in magenta), $n=3.375\left(1.5^{3}\right)$ (in blue) and $p=8\left(2^{3}\right)$ (in green) respectively, such that the three rectangles are resembled, and their sizes satisfy the ratio $1: 1.5: 2$.

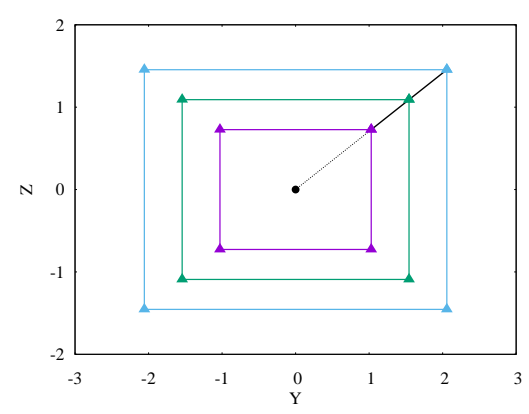

Figure 3: Two kinds of formation flying configurations with multiple spacecraft (marked with triangles), using the equilibria $1_{N}$ as nominal locations, which are all on the $Y-Z$ plane. The four magenta triangles denote the spacecraft with charge $q^{\star}$, while the blue and the green ones have charge $3.375 q^{\star}$ and $8 q^{\star}$, respectively. The black point at the origin corresponds to the leader, while the segments display the adjacent follower spacecraft. The first configuration consists of three resemble rectangles, while the second configuration is constructed with three spacecrafts with different charges, that are located along the black line going from the origin to the equilibrium point $1_{N}$ (with $Y>0$ and $Z>0$ ).

As stated in [4], we remember that the linear stability of the equilibria is only affected by the angular parameter $\beta$. Fig. 4 shows the behaviour, as a function of $\beta$, of the six eigenvalues associated to the equilibrium point $3_{R}$. It follows that the two $3_{R}$ with $(X=Z)$ are totally elliptic for $\beta \in(-\infty,-3.4525) \cup(0.9516, \infty)$. For a detailed stability analysis of other equilibrium points see [4]. 

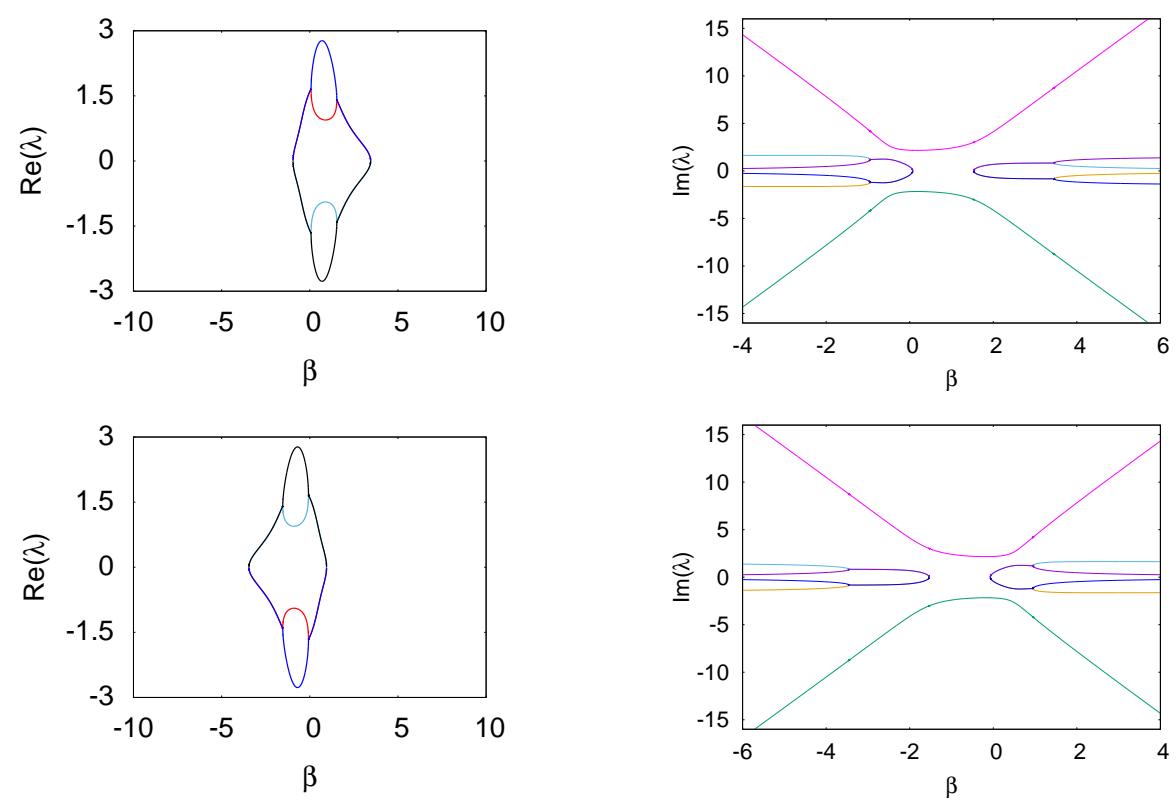

Figure 4: Behaviour, as a function of $\beta$, of the real part (left) and the imaginary part (right) of the eigenvalues associated to the equilibria $3_{R}$ with $Z=X$ (top), and $3_{R}$ with $Z=-X$ (bottom) [4]. Only the non-zero eigenvalues are shown.

We have chosen $\beta=10$ to explore the evolution of the green rectangular configuration shown in Fig. 2 which locates each spacecraft at one of the four $3_{R}$ equilibria, all of them on the $Y=0$ plane. According to their stability (Fig. 1), the four equilibrium points $3_{R}$ are the most stable ones (with six purely imaginary eigenvalues) for this value of $\beta$.

When the four satellites are relatively close to each other, and when comparing their mutual distances with respect to the distance to a tracking station, it is reasonable to assume that the tracking errors are the same for all them. We have considered an uncertainty in the position and velocity vectors of $\Delta \boldsymbol{X}=(1,0,1,1,0,1)^{\top} \times 10^{-3}$ adimensional units. With this uncertainty, the satellites no longer stay at the equilibrium points but move along invariant tori of very small size around the equilibria, as is shown in Fig. 5. If only the positions are perturbed $\left(\Delta \boldsymbol{X}=(1,0,1,0,0,0)^{\top} \times 10^{-3}\right)$, the results are similar, and the trajectories followed by the spacecraft are on slightly smaller tori than 


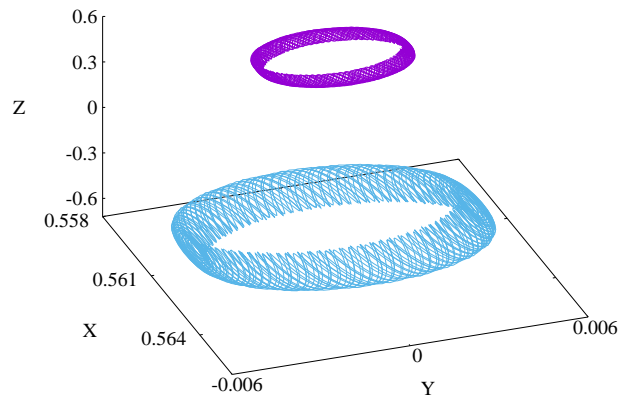

Figure 5: For $\beta=10$, trajectories of satellites located at two of the four $3_{R}$ equilibria (the ones with $X>0)$ when they are slightly displaced from their location $\left(\Delta \boldsymbol{X}=(1,0,1,1,0,1)^{\top} \times\right.$ $\left.10^{-3}\right)$. The results for the other two $3_{R}$ equilibria $(X<0)$ are symmetric w.r.t. the origin.

the ones obtained when both positions and velocities are perturbed. From now on we will take $\Delta \boldsymbol{X}=(1,0,1,1,0,1)^{\top} \times 10^{-3}$.

Due to the neutral stability of the $3_{R}$ equilibria with $\beta=10$, the invariant tori are bounded in a region whose size is of the same order as the perturbation $\Delta \boldsymbol{X}$, that is, $1 \times 10^{-3}$. A short-time time evolution of the four $3_{R}$ points is shown in Fig. 6. Clearly, the tight rectangular configuration no longer subsists, however, considering that the deviation is of the order of $1 \times 10^{-3}$, we can conclude that the configuration is not completely destroyed.

Fig. 7 displays the evolution of the relative distances between any two satellites of the above formation for a very long time interval, $\tau \in[0,1000]$. This is, for 1000 orbital periods of the leader satellite around the Earth. From the figure, it follows that the mutual distances display a quasi-periodic behaviour, and the distances between adjacent satellites fluctuate within the interval [1.115 : 1.13]. So the deviations in distance are one order of magnitude larger than the size of the perturbation. Note also that the distance between non-adjacent satellites is almost constant, in fact, of the order of $1 \times 10^{-4}$, which is one order of magnitude smaller than the one of the perturbation. In all the figures the satellites are always labelled as $S 1$ (purple), $S 2$ (blue), $S 3$ (brown) and $S 4$ (green) and sequenced in a counter-clockwise manner along the rectangle. 


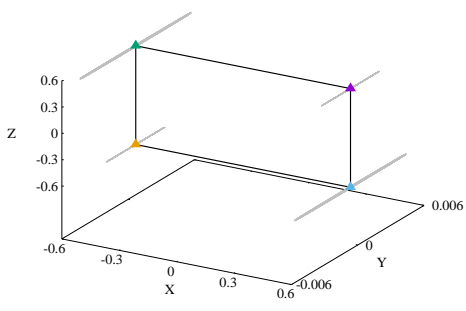

$t_{1}$

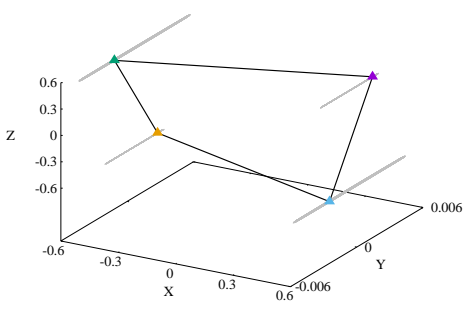

$t_{3}$

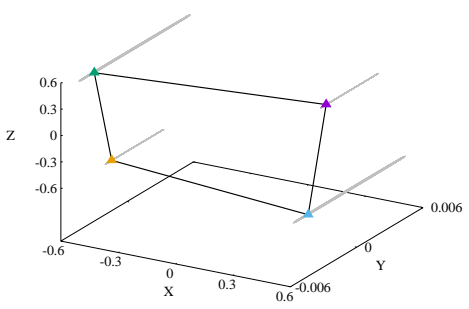

$t_{5}$

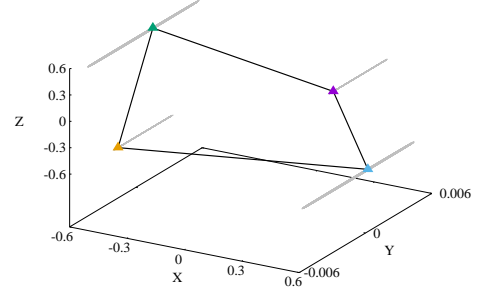

$t_{2}$

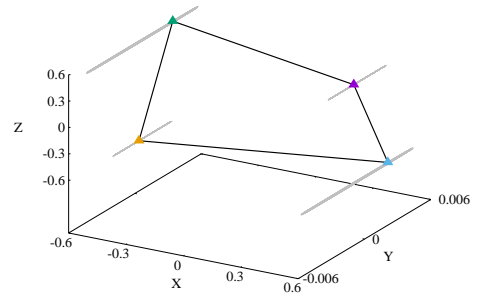

$t_{4}$

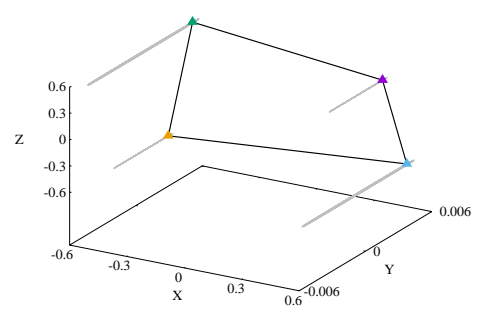

$t_{6}$

Figure 6: Time evolution of a configuration with four satellites deployed at the four equilibria $3_{R}$ and under perturbation $\Delta \boldsymbol{X}=(1,0,1,1,0,1)^{\top} \times 10^{-3}$. The six configurations displayed correspond to the epochs $t_{i}=0,80,160,240,320,400$ adimensional time-units. The grey segments are the orbits of the spacecraft along the invariant tori around the $3_{R}$ points, and the black segments connect adjacent satellites (marked as triangles). The colour code for the spacecraft is: $S 1$ in purple, $S 2$ in blue, $S 3$ in brown, and $S 4$ in green. 

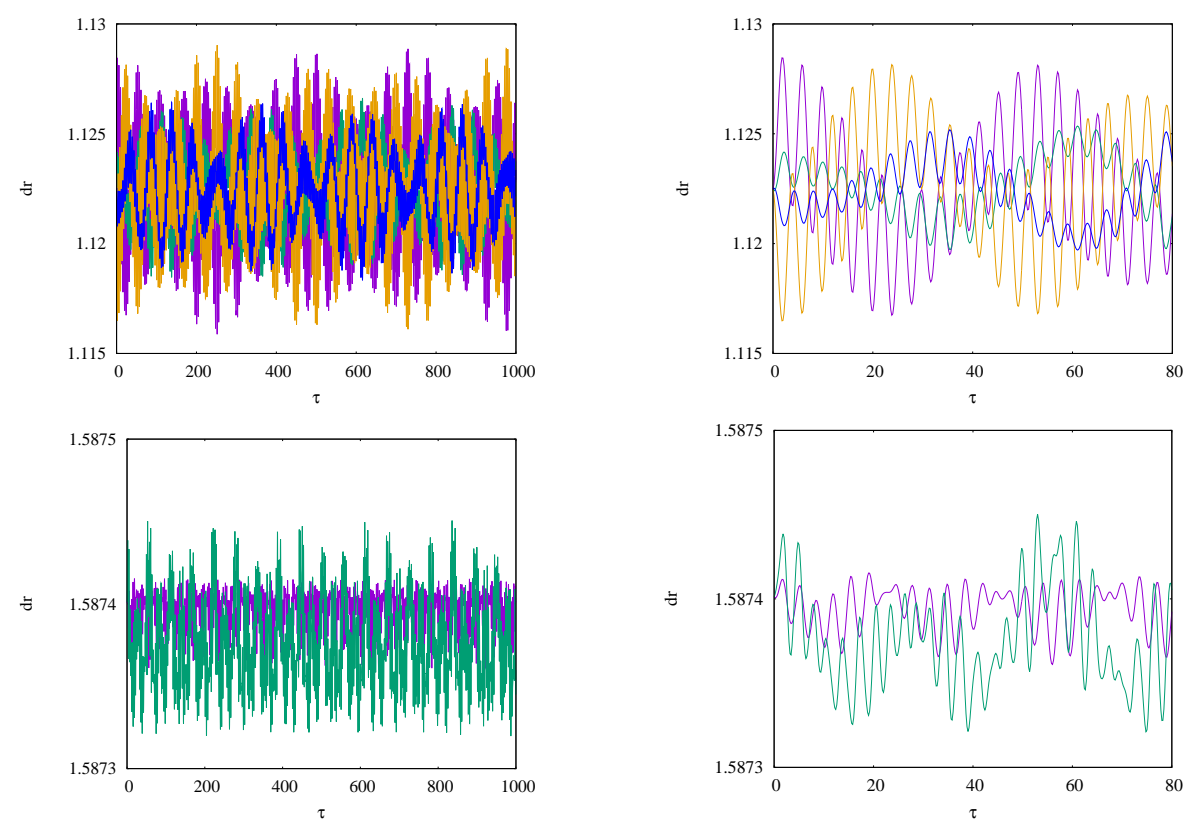

Figure 7: Evolution of the relative distance between any two satellites for $\tau \in[0,1000]$ (left column) and $\tau \in[0: 80]$ (right column). The top row displays the evolution of the distances between adjacent satellites: $S 1-S 2$ in purple, $S 2-S 3$ in green, $S 3-S 4$ in brown, and $S 4-S 1$ in blue. The bottom row show the evolution of the distances $S 1-S 3$ in purple, and $S 2-S 4$ in green. The colour code for the spacecraft is the same as in Fig. 6 .

The time evolution of the distances from the satellites, moving along the invariant tori, to the equilibrium point is shown in Fig. 8 for $\tau \in[0,1000]$. One can see that distances display a quasi-periodic behaviour, since the spacecraft are moving on a torus, and that the deviation is of the order of the perturbation $1 \times 10^{-3}$, which is the size of the torus. Moreover, the two curves corresponding to $S 1$ and $S 3$ almost overlap, and the same happens with the ones related with $S 2$ and $S 4$. This is because the two equilibria $3_{R}$ with $Z=\sqrt{X}$ have the same stability behaviour and are symmetric w.r.t. the origin, while the other two points with $Z=-\sqrt{X}$ have slightly different stability behaviour, as is shown in Fig. 4 .

We remark that, under the perturbation, the four satellites remain almost 

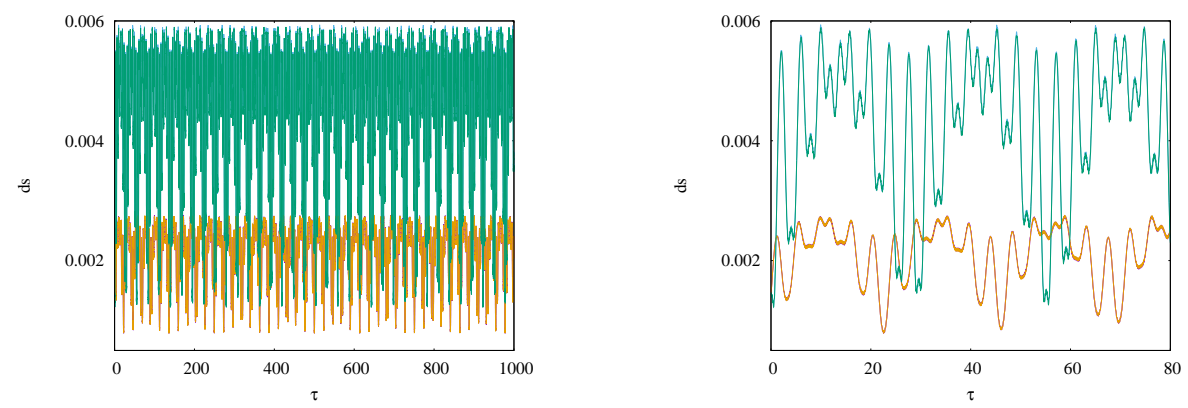

Figure 8: Time evolution of the distance from each satellite to the associated equilibrium point. The right hand side plot is a magnification for $\tau \in[0,80]$. The colour code for the spacecraft is the same as in Fig. 6 .

on the same plane. Fig. 9 shows the evolution with time of the angle between the normals to the planes defined by $S 1-S 2-S 3$ and $S 1-S 2-S 4$. One can see that this angle is always very small, less than $0.8^{\circ} \approx 0.014 \mathrm{rad}$. The angle between the $Y$-axis and the normal to the $S 1-S 2-S 3$ plane is also displayed in Fig. 9 and, as it follows from this figure, it is almost always aligned with the $Y$-axis (with a deviation less than $0.6^{\circ}$ ).
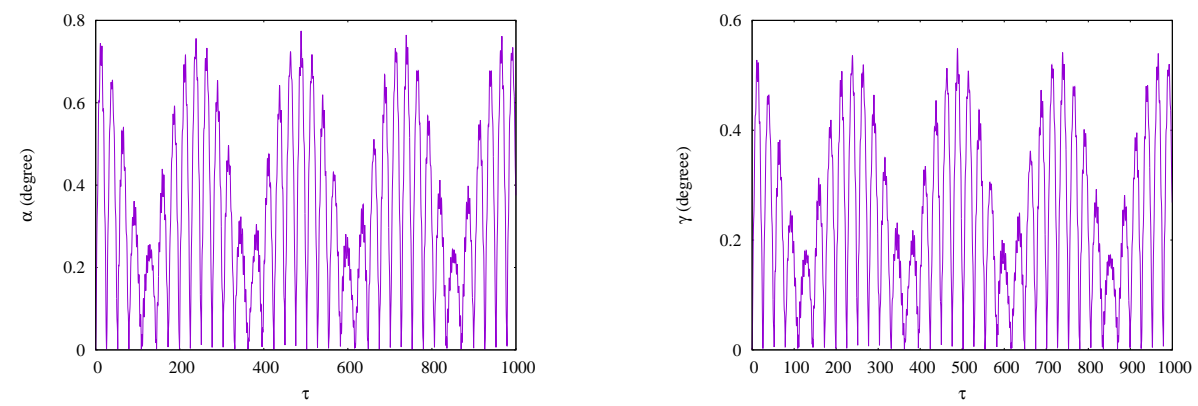

Figure 9: Left plot: Time evolution of the angle between the normal to the $S 1-S 2-S 3$-plane and the one to the $S 1-S 2-S 4$-plane. Right plot: time evolution of the angle between the normal to the $S 1-S 2-S 3$-plane and the $Y$-axis.

The other kinds of equilibrium points, except $3_{R}$, are always hyperbolic for all values of $\beta$ (see [4] for details). This means there will always be eigenvalues with non-zero real part. Due to this hyperbolic component, the spacecraft 
departing from those reference equilibria will escape soon even with very small initial displacement, as it will be seen in Fig. 10. This is a case for which control strategies are required to keep a formation using the equilibria as nominal locations for the spacecraft; it will be studied in the following section. Another case where formation keeping control has to be used is when the initial error is large, in this case the spacecraft will escape, even if the reference equilibrium point is totally elliptic.

\section{Formation keeping at the equilibrium points using a linear feedback control}

In this section we study the formation keeping using the charge $q$ as the only control parameter, this is: the self-maintenance capability of the formation configuration. The study is done assuming that the spacecraft of the formation can be controlled independently, which means that, in principle, different spacecrafts would require different controls.

\subsection{Controllability of the linearised system and the LQR feedback control law}

We denote the state vector of the follower by $\boldsymbol{X}=\left(X, Y, Z, X^{\prime}, Y^{\prime}, Z^{\prime}\right)^{\top}$, and study the controllability, under small perturbations, of the linearised system about the equilibrium point, which is given by:

$$
\Delta \boldsymbol{X}^{\prime}=\boldsymbol{A} \Delta \boldsymbol{X}+\boldsymbol{B}_{q}\left(\frac{q}{q^{*}}-1\right)
$$

where $\Delta \boldsymbol{X}=\left(\boldsymbol{X}-\boldsymbol{X}^{*}\right)$ is the small perturbation from the reference equilibrium point, whose coordinates are $X^{\star}=\left(X^{\star}, Y^{\star}, Z^{\star}, X^{\prime \star}, Y^{\prime \star}, Z^{\prime \star}\right)^{\top}$, and $q^{\star}$ is the nominal charge of the follower spacecraft; the $6 \times 6$ matrix $\boldsymbol{A}$ and the row vector $\boldsymbol{B}_{q}$ are given in the Appendix.

We note that in this paper the charge $q$ is treated equivalently to the chargeto-mass ration $q / m$, which is more commonly used when referring to the spacecraft surface charge. This is reasonable since the mass $m$ of the spacecraft is assumed to be constant when the propellent-less Lorentz force is used for actuation. 
The controllability matrix of the linearised system is:

$$
\boldsymbol{C}=\left[\begin{array}{llllll}
\boldsymbol{A} & \boldsymbol{A} \boldsymbol{B}_{q} & \boldsymbol{A}^{2} \boldsymbol{B}_{q} & \boldsymbol{A}^{3} \boldsymbol{B}_{q} & \boldsymbol{A}^{4} \boldsymbol{B}_{q} & \boldsymbol{A}^{5} \boldsymbol{B}_{q}
\end{array}\right] .
$$

Using the Matlab function "ctrb", for the computation of the rank of the matrix $\boldsymbol{C}$, it follows that it is maximal (equal to 6) for all the equilibrium points, except for the two points $3_{N}$, located along the $X$-axis. These points have the same location as the equilibrium points $2_{T}$ of the tangential case (see Table 2), which are linearly controllable, since in this case the rank of $\boldsymbol{C}$ is maximal. Therefore, the control of a formation using these locations for the spacecraft could only be achieved in the tangential case, and so only the points $1_{T}, 2_{T}$, and $3_{T}$ can be used.

Using the charge as the sole control input, a linear quadratic regulator is designed for the formation keeping at the equilibrium points, the performance index to be minimized is given by:

$$
J=\frac{1}{2} \int_{0}^{\infty}\left[\left(\boldsymbol{X}(\tau)-\boldsymbol{X}^{*}\right)^{T} \boldsymbol{Q}\left(\boldsymbol{X}(\tau)-\boldsymbol{X}^{*}\right)+R u^{2}\right] d \tau,
$$

where $u=q / q^{\star}-1$ is the adimensional control charge, $\boldsymbol{Q}$ is a $6 \times 6$ semipositive symmetric matrix, that penalizes the state error, while $R$ is a positive real number that penalizes the variations of the charge. The performance of the LQR can be tuned by varying carefully the values of $\boldsymbol{Q}$ and $R$. Without losing generality, we have used as initial guess $\boldsymbol{Q}=1000 \cdot \boldsymbol{I}$, where $\boldsymbol{I}$ is the $6 \times 6$ identity matrix, and $R=1$. Their values will be adjusted during the simulation according to the actual performance of the feedback control.

To minimize the performance index given by Eq. (8), the optimal control is given by:

$$
u(\tau)=-\boldsymbol{K}^{*}(\tau)\left(\boldsymbol{X}(\tau)-\boldsymbol{X}^{*}\right),
$$

where $\boldsymbol{K}$ is the 6 -dimensional feedback gain vector given by

$$
\boldsymbol{K}(\tau)=R^{-1} \boldsymbol{B}_{q}^{\top} \boldsymbol{P}(\tau)
$$

and where $\boldsymbol{P}(\tau)$ is the solution of the Riccati equation

$$
\boldsymbol{P}(\tau) \boldsymbol{A}+\boldsymbol{A}^{\top} \boldsymbol{P}(\tau)+\boldsymbol{Q}-\boldsymbol{P}(\tau) \boldsymbol{B}_{q} \boldsymbol{R}^{-1} \boldsymbol{B}_{q}^{\top} \boldsymbol{P}(\tau)=0 .
$$


The Matlab function 'lqr' has been used to compute the feedback gain vector $\boldsymbol{K}$, which is actually constant since the linearised system (6) is time invariant with constant coefficients $\boldsymbol{A}$ and $\boldsymbol{B}_{q}$. Then, we have $\boldsymbol{P}(\tau)$ also time independent.

According to the current technical limitations of the spacecraft surface charging, the bound of the total charge has been set to $[-10,10] \cdot q^{\star}$ in the following simulations. The total charge of the spacecraft, which is the sum of the control and the nominal charge, is given by:

$$
q(\tau)=(u(\tau)+1) \cdot q^{\star}
$$

where the control charge $u(\tau)$ is given by Eq. (9). We also remark that $u(\tau)$ should be constrained to make sure the total charge $q(\tau)$ satisfies the prescribed bound $[-10,10] \cdot q^{\star}$, by the following rule:

$$
\left\{\begin{array}{cl}
u(\tau)=-11, & \text { if } u(\tau)<-11 \\
u(\tau)=9, & \text { if } u(\tau)>9
\end{array}\right.
$$

\subsection{Formation keeping of single follower configuration}

We first consider the leader-follower configuration, where only one single follower is included. If the follower spacecraft is located at an unstable equilibrium point, it will escape from it even with a very small perturbation. As an example, the dot-dashed line in Fig. 10 shows the propagation, in the non-linear system defined by Eq. (3) in the radial case, of the perturbed equilibrium point $3_{R}$ (with $X>0$ and $Z>0)$ when the perturbation $(1,0,1,1,0,1)^{\top} \times 10^{-3}$ is added. The value of the ratio between the mean motion of the leader around the Earth and the angular velocity of the dipole, $\beta$, in (5) is one for this simulation.

For this same value of $\beta$, and departing from the same perturbed initial condition, Fig. 10 displays the results of the linear feedback control, defined by Eq. (9), using $q$ as the unique control parameter.With $\beta=1$, the values of $\boldsymbol{A}$ 

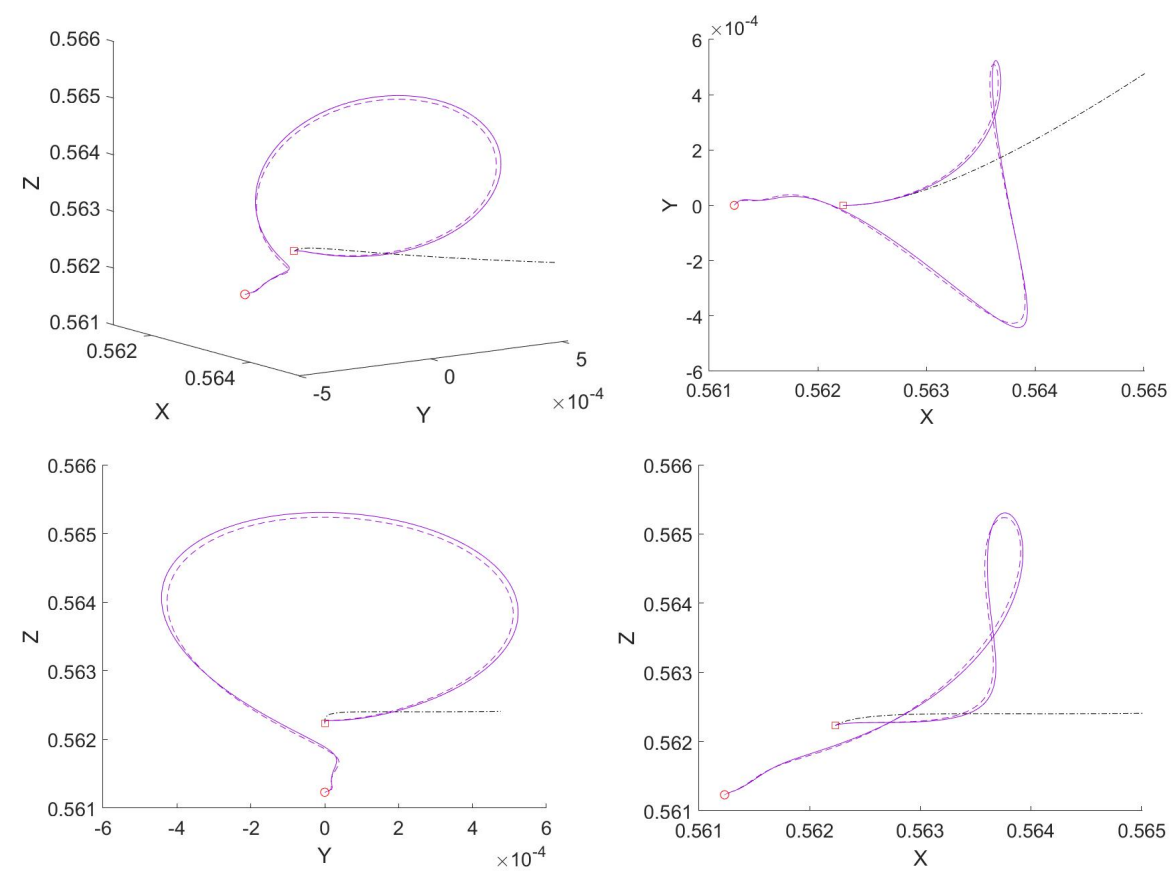

Figure 10: 3D representation (top left) and $X-Y$ (top right), $Y-Z$ (bottom left), $X-Z$ (bottom right) coordinate projections of trajectories departing from the $3_{R}$ equilibrium $(X>0, Z>0)$ with a displacement equal to $(1,0,1,1,0,1)^{\top} \times 10^{-3}$ (red square). The black dot-dashed line, which deviates very fast from the initial condition, is the radio-controlled trajectory; the dashed line is the controlled trajectory of the linearised system defined by (6), and the solid line the controlled trajectory of the non-linear system defined by (3) and (5) in the radial case, with $\beta=1$.

and $\boldsymbol{B}_{q}$ in the linearised system (6) are:

$$
\boldsymbol{A}=\left[\begin{array}{cccccc}
0 & 0 & 0 & 1 & 0 & 0 \\
0 & 0 & 0 & 0 & 1 & 0 \\
0 & 0 & 0 & 0 & 0 & 1 \\
7.5 & 0 & 1.5 & 0 & -1 & 0 \\
0 & 1 & 0 & 1 & 0 & -1 \\
1.5 & 0 & -4.5 & 0 & 1 & 0
\end{array}\right], \quad \boldsymbol{B}_{q}=\left[\begin{array}{c}
0 \\
0 \\
0 \\
-1.6837 \\
0 \\
0.5612
\end{array}\right] .
$$

and the feedback gain vector is $\boldsymbol{K}=(114.69,-147.24,-113.07,0.62,-138.13,22.82)$.

As it is shown in the same figure, one can see that the follower spacecraft 
escapes fast from the initial condition when no control is applied. If the control is on, then it requires less than 10 time units (10 times the leader's orbital period) to return to the nominal location, which is set at the $3_{R}$ point. Moreover, the controlled trajectories both in the linearised system 6 and in the non-linear one 3 are very close.
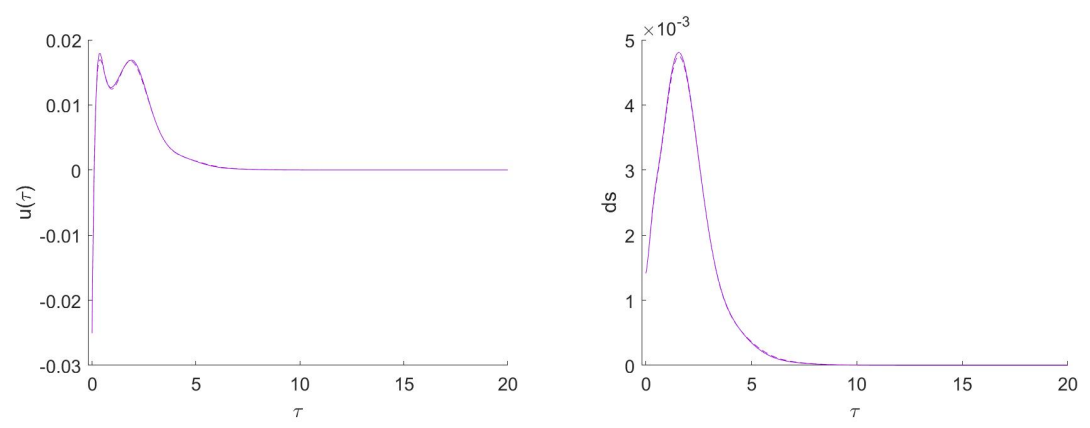

Figure 11: Time evolution of the control input $u(\tau)$ (left), and distance of the spacecraft to the target equilibrium point $3_{R}$ (right). The dashed line corresponds to the simulation in linearised system, while the solid line to the one in the non-linear system, with $\beta=1$.

The time history of the control charge $u(\tau)$, and the distance to the target point $3_{R}$, are displayed in Fig. 11. The curves of the controlled linear and nonlinear systems nearly coincide. This is because the non-linear terms are very small due to the small initial error considered (of the order of 0.001), and the maximum deviation of the spacecraft from its target less than 0.005 . Note that the unit of the charge control is $q^{\star}$, and the total charge variation required for the control is less than $3 \%$ of the nominal charge $q^{\star}$.

\subsection{Formation keeping of a multiple-follower configuration with spacecraft of} same charge

We consider now a configuration with four satellites, each one set at one of the four $3_{R}$ equilibria, this is, the square formation on the green plane displayed in Fig. 2. The four $3_{R}$ equilibria are labelled as: $3_{R}^{1}(X>0, Z>0), 3_{R}^{2}$ $(X>0, Z<0), 3_{R}^{3}(X<0, Z<0)$, and $3_{R}^{4}(X<0, Z>0)$. The feedback gain 
for each equilibrium, with $Q=10 \cdot \boldsymbol{I}$ and $R=1$, is:

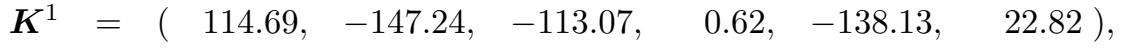

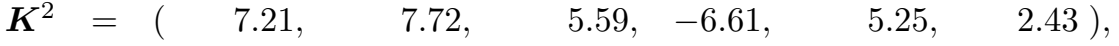

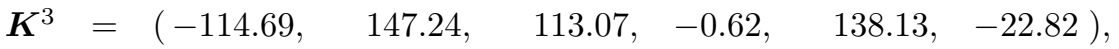

$$
\begin{aligned}
& \boldsymbol{K}^{4}=\left(\begin{array}{llllll}
-7.21, & -7.72, & -5.59, & 6.61, & -5.25, & -2.43
\end{array}\right) .
\end{aligned}
$$

Note that the gains of the two equilibria with $X=Z,\left(3_{R}^{1}\right.$ and $\left.3_{R}^{3}\right)$, and $\left(3_{R}^{2}\right.$ and $3_{R}^{4}$ ), which are symmetric with respect to the origin, are the opposite of each other. This is: $\boldsymbol{K}^{1}=-\boldsymbol{K}^{3}$, and $\boldsymbol{K}^{2}=-\boldsymbol{K}^{4}$, while they are different from the two equilibria with $X=-Z$. This fact is coherent with the stability of the four equilibria $3_{R}$ (see Fig. 4 ). The same symmetry can also be observed in the trajectories of the satellites when they are set at the displaced equilibria.

Each satellite is controlled changing its own charge $q$. Taking the same value of $\boldsymbol{Q}$ and $R$ as in Section 4.2, as well as the same initial displacement, $(1,0,1,1,0,1) \times 10^{-3}$, for the four satellites, Fig. 12 shows the time history of the control input $u(\tau)$, and the distance of each spacecraft to its target equilibrium point.
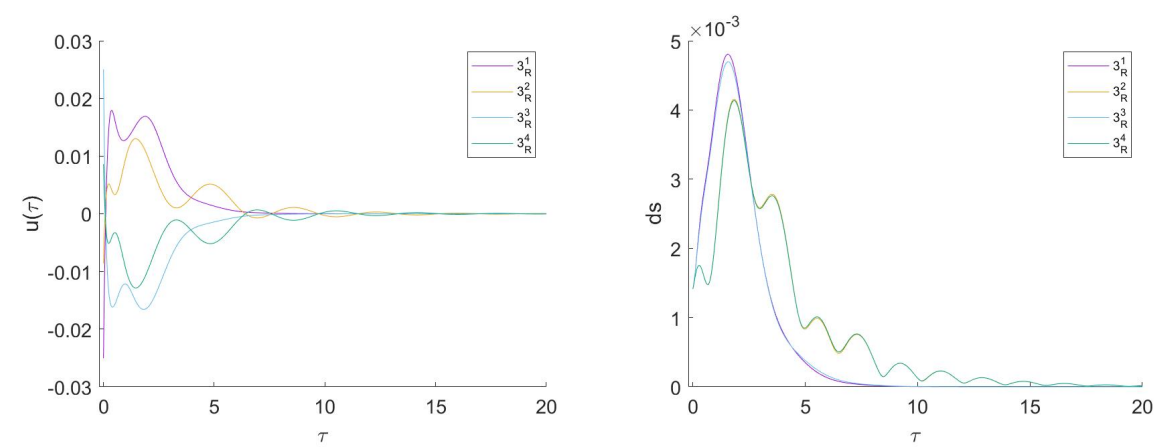

Figure 12: Time evolution of the control inputs $u(\tau)$ (left), and distance of the spacecraft to their target $3_{R}$ equilibrium points (right). The colour code for the equilibria is: $3_{R}^{1}$ green, $3_{R}^{2}$ brown, $3_{R}^{3}$ orange, and $3_{R}^{4}$ purple. The computations are done in the non-linear system defined by (3) and (5), with $\beta=1$.

From Fig. 12 it follows that the controls have a nearly symmetric evolution when the equilibria are symmetric with respect to the origin, this is for $3_{R}^{1}$ 
and $3_{R}^{3}$, and for $3_{R}^{2}$ and $3_{R}^{4}$. The fact that the two curves giving the distance associated to $3_{R}^{1}$ and $3_{R}^{3}$ do not coincide is because their initial states are not symmetric with respect to the origin. Note that in all the cases the total charge variation required for the control is about $3 \%$ of the nominal charge $q^{\star}$, and that the charge control of the two $3_{R}$ equilibria with $X=Z$ is slightly larger than the one required for the two with $X=-Z$. This is due to the instability (given by the value of their real eigenvalues shown in Fig. (4) of $3_{R}^{1}$ and $3_{R}^{3}$ is slightly larger than the one of $3_{R}^{2}$ and $3_{R}^{4}$. We also remark that for all the spacecraft their maximum deviation from their target point is of the order of the initial displacement $\left(1 \times 10^{-3}\right)$. Moreover, the trajectories of the linear and non-linear systems are quite close to each other. We remark that the controllability, using the LQR control law given by Eq. (9), with $q$ as the unique control parameter, cannot be achieved when the initial displacement is of the order $1 \times 10^{-2}$ (this is, one order of magnitude larger than the one shown in Fig. 10); in this case the controlled trajectories escape fast due to the strong instability caused by the large saddle component of the equilibrium point.

When the value of $\beta$ is increased, the equilibrium points become more stable. Table 3 lists the three pairs of eigenvalues associated to the equilibria $3_{R}$ with $\beta=1,2,10$. It can be seen that the topological type of the two equilibria $3_{R}$ with $X=Z$ goes from saddle $\times$ saddle $\times$ centre (for $\beta=1$ ), to complex saddle $\times$ centre (for $\beta=2$ ), and to centre $\times$ centre $\times$ centre, (for $\beta=10$ ), while the other $3_{R}$ equilibria with $X=-Z$ are always totally elliptic, this is: centre $\times$ centre $\times$ centre.

When $\beta=2$, the instability of the $3_{R}^{1,3}$ equilibria is very mild (the modulus of the saddle eigenvalues is small) and controllability can be achieved even for an initial displacement from the equilibrium point as large as $(1,0,1,1,0,1)^{\top} \times$ $10^{-1}$. As Fig. 13 shows, the maximum charge variation required for the control is of the same order of the nominal charge $\left(<-1.2 q^{\star}\right)$, and the maximum distance of the spacecraft to the target equilibrium point is of the order of the initial error $(\approx 0.25)$. In all cases, the trajectories of the linear and non-linear systems are relatively close to each other, due to the fact that the two equilibria $3_{R}^{1,3}$ 
Table 3: Eigenvalues associated to the equilibria $3_{R}$ for different values of $\beta$.

\begin{tabular}{ccccc}
\hline $3_{R}$ & $\beta$ & $\lambda^{1,2}$ & $\lambda^{3,4}$ & $\lambda^{5,6}$ \\
\hline & 1 & \pm 2.6263 & \pm 0.9488 & $\pm 2.4079 i$ \\
$3_{R}^{1,3}$ & 2 & $0.9895 \pm 0.6898 i$ & $-0.9895 \pm 0.6898 i$ & $\pm 4.1239 i$ \\
$(X=Z)$ & 10 & $\pm 29.6282 i$ & $\pm 1.4666 i$ & $\pm 0.1381 i$ \\
\hline & 1 & $\pm 4.3723 i$ & $\pm 1.3723 i$ & $\pm i$ \\
$3_{R}^{2,4}$ & 2 & $\pm 7.8175 i$ & $\pm 1.6327 i$ & $\pm 0.4701 i$ \\
$(X=-Z)$ & 10 & $\pm 33.4281 i$ & $\pm 1.5973 i$ & $\pm 0.1124 i$ \\
\hline
\end{tabular}

are hyperbolic, and the stability behaviour of the linear and non-linear system resemble each other very well. We also remark that the rate of convergence to the equilibrium points of the perturbed trajectories mainly depends on the stability of the equilibria. As it is seen in Fig. 13, the two trajectories (of the linear and non-linear systems) associated to $3_{R}^{1,3}$ converge to the equilibrium point almost simultaneously; the same phenomena can be seen in Fig. 13 for $\beta=10$ and an initial displacement $(1,0,1,1,0,1)^{\top} \times 10^{-2}$. Although in this latter case the convergence is not so fast, it takes about 12 time units to the distance the equilibrium point to become almost zero, in front of the 8 time units required for $\beta=2$ and a displacement equal to $(1,0,1,1,0,1)^{\top} \times 10^{-2}$.

For the other two equilibria $3_{R}^{2,4}(X=-Z)$ the behaviour of the control is different. Now the equilibria are purely elliptic (they have three pairs of purely imaginary eigenvalues). The ellipticity leads to a very weak controllability using the linear feedback control law. For beta = 2, Fig. 13 shows the time evolution of the control charge, and distance to the target equilibrium points. One can see that for these target points the spacecraft oscillate around the equilibrium points during a long-time interval without converging to them. The displacement of the spacecraft from the target equilibria is still of the order of $1 \times 10^{-5}$, even after 200 time-units, with a maximum distance $(\approx 1)$ about 3 times larger than that of the two equilibria $3_{R}^{1,3}$. The required charge control also exhibits an osculating behaviour, with a maximum as large as $-5.2 q^{\star}$, this is: 5.2 times the 

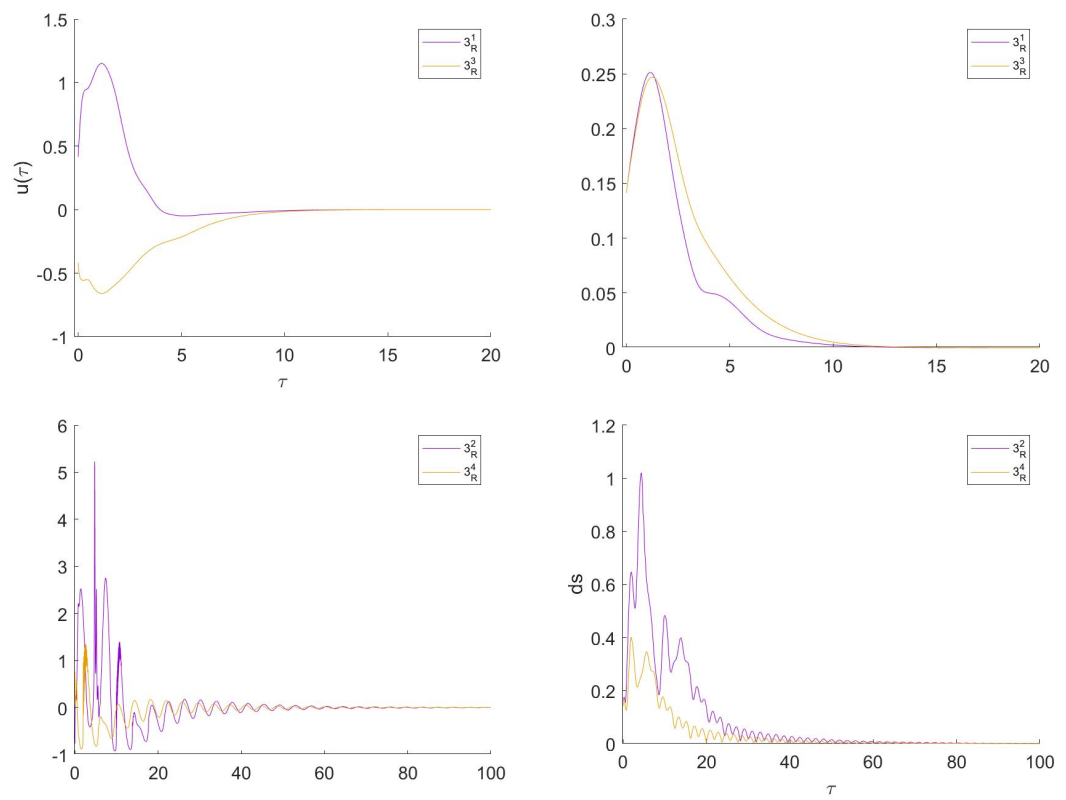

Figure 13: Time evolution of the control input $u(\tau)$ (left), and distance of the spacecraft to its target equilibrium point (right). The top row corresponds to the equilibrium points $3_{R}^{1,3}$, and the bottom row to $3_{R}^{2,4}$. The computations are performed in the non-linear system defined by (3) and (5), with $\beta=2$ and with an initial displacement of the spacecraft equal to $(1,0,1,1,0,1)^{\top} \times 10^{-1}$.

nominal charge. We can conclude that, in these cases, the rate of convergence of the control procedure is too slow for practical applications.

Fig. 14 displays the trajectories of the spacecraft departing from the four equilibria $3_{R}$ with the same initial error $(1,0,1,1,0,1)^{\top} \times 10^{-1}$ and the same value of $\beta=2$. From this figure it follows that for the two hyperbolic equilibria $\left(3_{R}^{1}\right.$ and $\left.3_{R}^{3}\right)$ the controlled trajectories of the linear and non-linear systems are not too far from each other, and converge fast to their target equilibrium point. This can be explained by Hartman-Grobman theorem since, according to it, the linearised and non-linear system around a hyperbolic equilibrium point are topologically equivalent to each other. For the two elliptic equilibria $\left(3_{R}^{2}\right.$ and $3_{R}^{4}$ ), the controlled trajectories for the linear and non-linear systems differ very much from each other since the stability of the non-linear system cannot be 
represented by the linear one when the equilibria is totally elliptic. In this case, the non-linear terms may lead to growth or decay of the displacement from the equilibrium point [9].
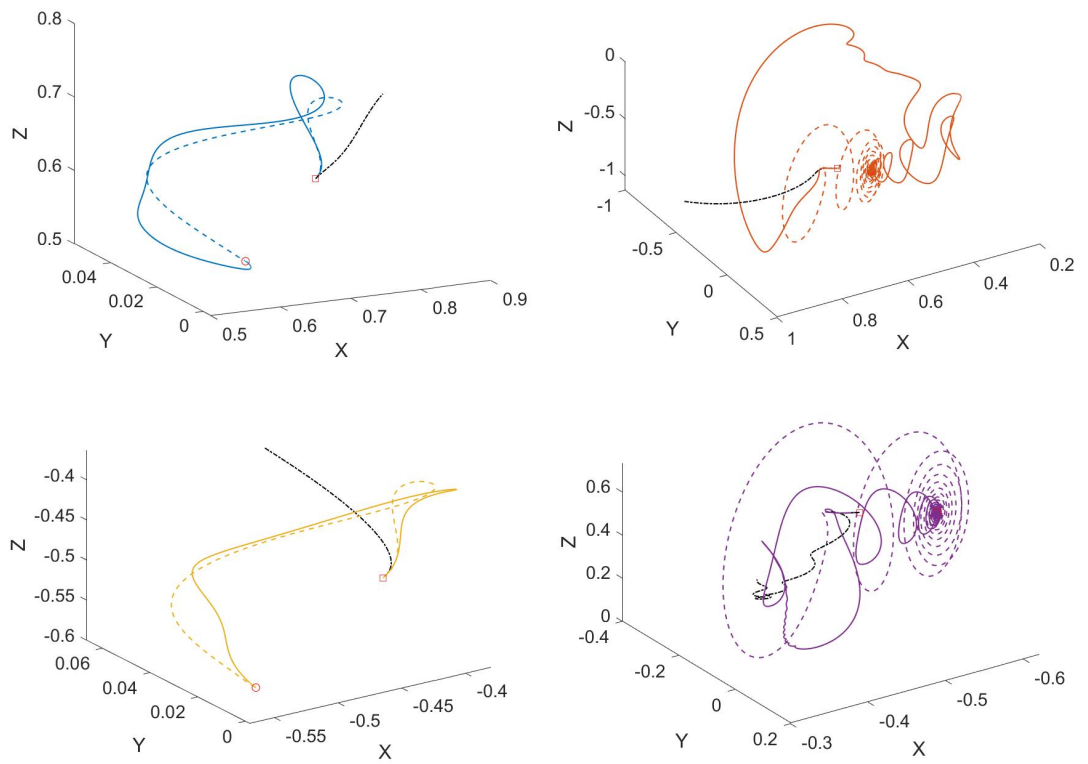

Figure 14: Trajectories departing from the equilibrium point $3_{R}^{1}$ (top left), $3_{R}^{2}$ (top right), $3_{R}^{3}$ (bottom left) and $3_{R}^{4}$ (bottom right), with $\beta=2$, and an initial displacement (square) equal to $(1,0,1,1,0,1) \times 10^{-1}$. The dot-dashed lines, which deviate from the initial conditions, are the non-controlled trajectories, the dashed lines correspond to the controlled ones of the linearised system defined by (6), and the solid lines are the controlled trajectories of the non-linear system defined by (3).

For the complex saddle $\times$ centre equilibrium point $3_{R}^{1}($ with $\beta=2)$, the displacement applied to the initial conditions can be increased up to a very large value, $(1,0,1,1,0,1)^{\top} \times 0.25$, without losing controllability when we take $\boldsymbol{Q}=10 \cdot \boldsymbol{I}$. These conditions are referred to as case 1. Fig. 15.(top) shows the trajectories of the spacecraft departing from $3_{R}^{1}$ with this error, obviously the controlled non-linear trajectory is far from the linear one, since most probably the linear approximation is no longer valid for these large deviations. The time evolutions of the corresponding control charge and displacement from the 
target point are shown in Fig. 16 as purple lines. In this case, the maximum distance to the target point is very large $(\approx 0.75)$, which is as large as the distance $(=0.7937)$ of $3_{R}^{1}$ from the origin (note that the location of $3_{R}^{1}$ is at $[0.5612,0,0.5612])$. We remark that the initial error is considered up to the second digit after the decimal, and the charge control reaches its lower bound $(-11)$ at $\tau=4.28$, as seen in Fig. 16 (purple). It must be noted that the controllability is lost with initial displacement larger than $(1,0,1,1,0,1)^{\top} \times 0.25$ if the charge control variations are limited to $[-11,9] \cdot q^{\star}$, such that the total charge is bounded within $[-10,10] \cdot q^{\star}$.

However, when we tune change the value of $\boldsymbol{Q}$ as in control Eq. (9), better controllability can be achieved when a larger initial error is considered. Fig. 15 (bottom line) shows also the trajectories of two other cases:

- case 2: $\boldsymbol{Q}=500 \cdot I$ and initial error $(1,0,1,1,0,1)^{\top} \times 0.25$;

- case 3: $\boldsymbol{Q}=500 \cdot I$ and initial error $(1,0,1,1,0,1)^{\top} \times 0.44$.

while the associated charge control and displacement are displayed in Fig. 16. It follows that the value of $\boldsymbol{Q}$ not only affects the rate of converge but also the controllability of the system. With the same initial error $\left((1,0,1,1,0,1)^{\top} \times 0.25\right)$, the controlled linear and non-linear trajectories are closer in case $2(\boldsymbol{Q}=500 \cdot I)$, which implies stronger controllability and rate of convergence. The maximum charge variation is also much smaller $\left(4.01 q^{\star}\right)$ than the one required in case 1 $-11 q^{\star}$, while the displacement is only $76 \%$ of the one in case 1 . With $Q=500 \cdot I$, the system shows extremely strong controllability, the maximum controlled error can be as large as $(1,0,1,1,0,1)^{\top} \times 0.44$, with the maximum deviation as far as 1.06 length units, much larger than the distance of the reference equilibrium point from the origin. We note that no larger initial error can be controlled when $\beta=2$, when the value of $Q$ has been optimized, since the required control charge reaches its upper bound in several intervals $(\tau \in[0,2])$, which implies that the charge, which is the unique control parameter, has reached its maximum, according to the considered bound constraint. 
Comparing these results of case 1 with the ones corresponding to a smaller initial displacement $\left((1,0,1,1,0,1)^{\top} \times 10^{-1}\right)$, and shown in Fig. 13, we can conclude that increasing the initial displacement slows down the rate of the convergence of the control method. Moreover, the required charge control variation increases $\left(-11 q^{\star}\right.$ vs $\left.-1.2 q^{\star}\right)$ when the initial error also does. The same phenomena can also be found when we compare the results of case 2 and case 3 (see Fig. 16).

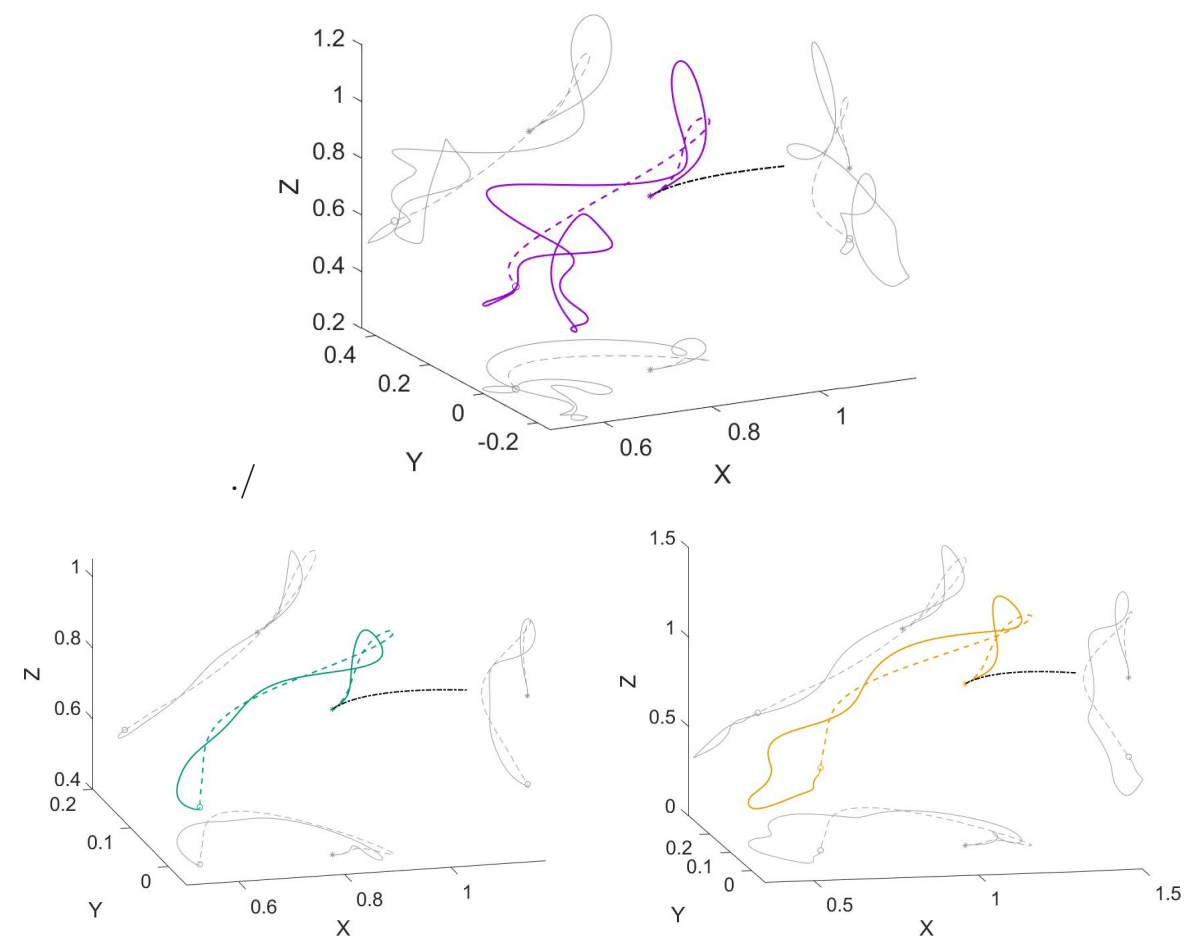

Figure 15: Uncontrolled trajectory (black dot dashed) and controlled one for a spacecraft using the linear (coloured dashed) and non-linear (coloured solid) systems, and coordinate projections (grey) of the controlled trajectories. The computations are performed with $\beta=$ 2 , and the initial errors departing from the equilibrium point $3_{R}^{1}$ (circle) and value of $\boldsymbol{Q}$ correspond to case 1 (top), case 2 (bottom left) and case 3 (bottom right).

According to Table 3, when $\beta=10$ the four $3_{R}$ equilibrium points are totally elliptic (all the eigenvalues are purely imaginary) and, as it was already 

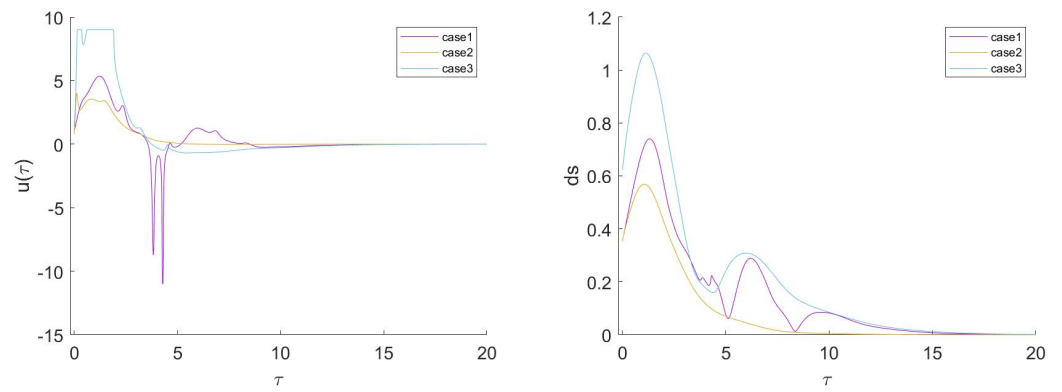

Figure 16: Time evolution of the control input $u(\tau)$ (left), and distance of the spacecraft to their target equilibrium points (right). Computations are performed in the non-linear system defined by (3) and (5), with $\beta=2$, and coloured according to the three defined condition cases: purple (case 1), brown (case 2) and cyan (case 3).

mentioned for the $3_{R}^{3,4}$ points with $\beta=2$, in this case it is difficult to maintain the configuration using only the charge as control parameter.

Figure 17 shows the results obtained for this value of $\beta$ with an initial displacement for all the spacecraft of $(1,0,1,1,0,1)^{\top} \times 10^{-2}$. In this example, the maximum charge variation required for the control of the spacecraft located at $3_{R}^{2}$ and $3_{R}^{4}$ is about four times larger than the one required for spacecraft located at $3_{R}^{1}$ and $3_{R}^{3}$.

It must be also mentioned that, even with an initial displacement of the order of 0.01 , the time required to reach the vicinity of the target equilibria is very large, about 80 time units.

For the other equilibrium points the controllability results are similar to the ones discussed for the $3_{R}$ points and mainly depend on the characteristics of their eigenvalues. We recall that all the other points are hyperbolic regardless of the value of $\beta$ so, for better controllability, the best values of $\beta$ correspond to those such that the associated eigenvalues have real as small as possible. In particular, in Fig. 18 we display the results of formations with two spacecraft deployed at each of the two $2_{T}$ points $\left(2_{T}^{1}\right.$ with $X>0$ and $2_{T}^{1}$ with $\left.X<0\right)$, initial conditions have been taken as $(1,0,1,1,0,1)^{\top} \times 10^{-1}$ for initial error, $\beta=1$ and $\boldsymbol{Q}=100 \cdot \boldsymbol{I}$. As stated in Section 4.1, these points are the only 

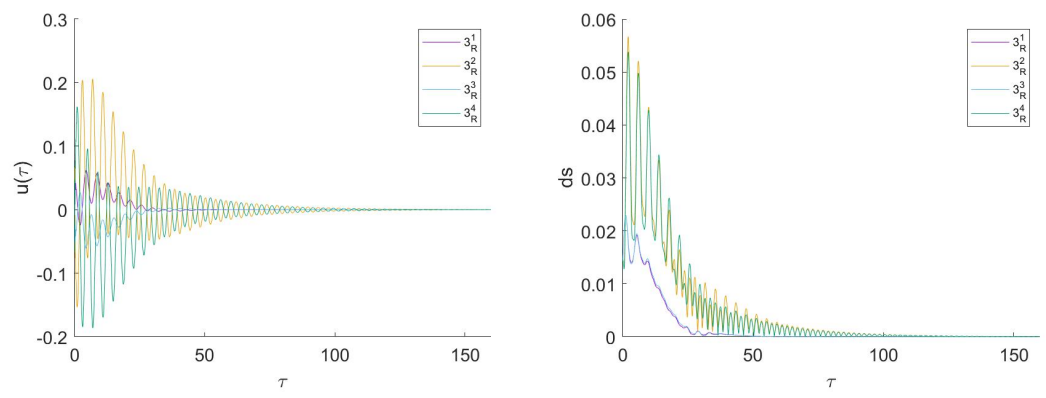

Figure 17: Time evolution of the control input $u(\tau)$ (left), and distance of the spacecraft to their target equilibrium points (right). Computations are performed in the non-linear system defined by (3) and (5), with $\beta=10$, and with an initial displacement for all the spacecraft of $(1,0,1,1,0,1)^{\top} \times 10^{-2}$. The colour code is the same one as in Fig. 12 .

option if the nominal locations of the $3_{N}$ points are required. We recall that the $2_{T}$ points (with the same location as the $3_{N}$ ) are controllable while the $3_{N}$ are not, as stated in Section 4.1 .

In Fig. 18 we notice the symmetry of the trajectories with respect to the $Y-Z$; control charges and deviations also exhibit symmetries similar to the ones in Fig. 12 .

\subsection{Formation keeping of a multiple-follower configuration with spacecraft of different charges}

The previous configurations are designed based on the assumption that the satellites are identical in mass and charge, so they have the same charge-to-mass ratio. If we adjust the charge in one or more satellites of the configuration, for instance by means of an electron beam, new equilibrium points are obtained along the same axis, but at different distances. As a consequence, once a simple configuration is designed in adimensional units, a similar configuration of different size, or a combination based on the original configuration of any number and any size can be easily obtained.

In this section we consider multi-follower configurations in which all the follower spacecraft have different charges. While the reference equilibrium point is the same, the charge defines the length unit, and so the real physical location 

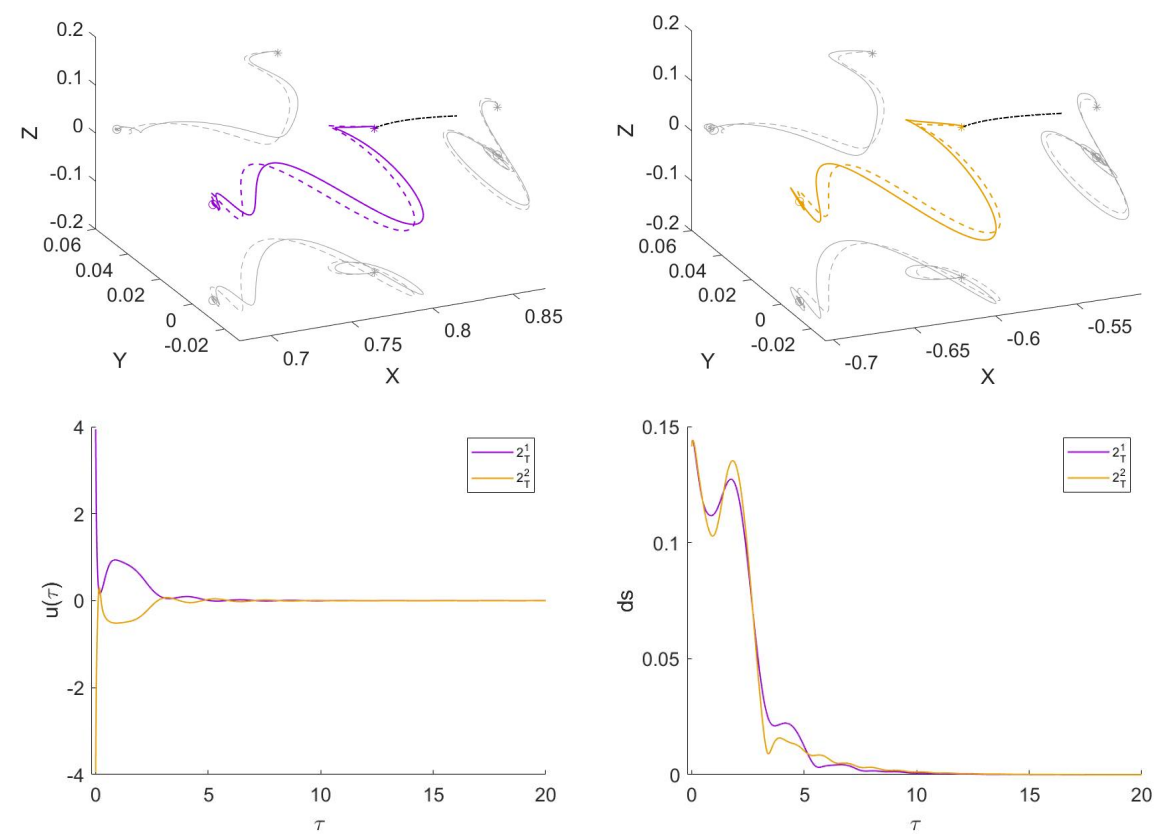

Figure 18: The trajectories of two spacecraft departing from the equilibrium point $2_{T}^{1}$ (top left) and $2_{T}^{1}$ (top right), both with a displacement equal to $(1,0,1,1,0,1)^{\top} \times 10^{-1}$ (circle), and the time evolution of the required control charge (bottom left) and deviation from the target $2_{T}$ (bottom right). The black dot dashed lines are the perturbed trajectory without control, while the controlled trajectories are shown in dashed lines (linear system defined by Eq. (6)) and solid lines (non-linear system defined by Eq. (3) in the tangential case, and in grey are the $2 \mathrm{D}$ coordinate projections of the controlled trajectories.

of the equilibrium points differ from each other. An example is the string configuration shown in Fig. 3, with the reference location on the line passing through the $1_{N}$ equilibrium point and the origin.

For simplicity, we introduce the charge ratio $\eta=\frac{q}{q^{\star}}$, where $q$ is the charge of the spacecraft and $q^{\star}$ is the reference nominal charge. Using the equilibrium point $3_{R}$ as nominal location, we design a configuration of three deputies with charges $q_{i}=\eta_{i} \cdot q^{\star}, i=1,2,3$ and $\eta_{1}=1, \eta_{2}=3.375, \eta_{3}=8$. Their nominal positions are given in Table 4 , where the length unit is chosen to be the same, that is $a^{\star}$, the one associated to $q^{\star}$. 
Table 4: The actual position of three deputies with different charges $\eta q^{\star}$ using $3_{R}^{1}$ as the nominal location.

\begin{tabular}{cccc}
\hline$\eta$ & $X$ & $Y$ & $Z$ \\
\hline 1 & 0.5612 & 0 & 0.5612 \\
3.375 & 0.8418 & 0 & 0.8418 \\
8 & 1.1225 & 0 & 1.1225 \\
\hline
\end{tabular}

We consider the same initial error $(1,0,1,1,0,1)^{\top} \times 0.05$, and $\boldsymbol{Q}=100 \cdot \boldsymbol{I}$, $R=1$ for all three spacecraft. During the simulations we have used different length units for different charges. Thus, all the equations can be kept the same without any modification, and the feedback gain vectors $\boldsymbol{K}$ are also the same regardless of the value of the charge; this is the advantage of using dimensional units. However, special attention must be paid to transform the initial error to the one corresponding to $q_{i}(i=1,2,3)$, which is $a^{\star} \cdot \eta^{\frac{1}{3}}$, before we start the propagation of the controlled trajectory. Also, the inverse transformation on position and velocity needs to be performed after the integration to obtain the results in the same units for comparison.

We remark that the control charge given by Eq. (9) has always the unit associated to $q_{i}$, and if the total charge $q(\tau)=(u+1) * \eta \cdot q^{\star}$ violates the bound $[-10,10] \cdot q^{\star}$, then the adimensional control charge is determined by the following rule:

$$
\left\{\begin{array}{cl}
u(\tau)=-10 / \eta-1, & \text { if }(u(\tau)+1) \cdot \eta<-10 \\
u(\tau)=10 / \eta-1, & \text { if }(u(\tau)+1) \cdot \eta>10
\end{array}\right.
$$

When the unit of charge is chosen to be the one of $q^{\star}$, the control and total charge is $u(\tau) \cdot \eta$ and $q(\tau)=(u(\tau)+1) \cdot \eta$, respectively. We remark that the controllability is strongly affected by the nominal charge $\eta \cdot q^{\star}$ of the spacecraft, as can be clearly seen from equation (14). A large value of $\eta$ leads to a small range of values of the control charge $u(\tau)$.

The trajectories of the linear and non-linear systems, as well as the time evolution of the control charge and displacement with respect to the reference 
equilibrium point, are shown in Fig. 19. In this figure we can see that, with same initial error of the order of 0.05 , the trajectories of the three spacecraft with different charges exhibit the same behaviour, and the curves of their deviations almost overlap each other. It is obvious that the controllability is not affected by the charge of spacecraft when the initial error is small. The rate of convergence is almost the same, due to the fact that the reference equilibrium point is the same one, and so is the stability, which plays the dominant role in controllability of the system. The different maximum values of control charge are caused by the initial errors; although they are the same in $a^{\star}$ units, they are different in $a^{\star} \cdot \eta^{\frac{1}{3}}$ units. Again, since the initial error is relatively small, the linear and nonlinear controlled trajectories are very close to each other. The spacecraft moves within the vicinity of its reference equilibrium point, with the displacement less than 0.12 , almost 2.5 times the initial error. Due to the large distance between the three nominal locations considered, there is no collision risk between the deputies.

However, as stated in Section. 4.3, when the initial error is increased, the trajectory, if still controllable without escaping, can depart at a large distance from the reference equilibrium point. This fact introduces a collision risk in the above string configuration, since the distance between two equilibria is a linear function of the cubic root of the charge ratio $\eta$, which could be small with the bound $\eta \in[-10,10]$.

To explore this case with collision risk we consider a large initial error for each spacecraft, while the values of $\boldsymbol{Q}$ are tuned for each spacecraft for the best control performance with $R=1$ (see Table 5 for the values of the related parameters). The values of the feedback gain vector $\boldsymbol{K}$ are the same for charges $q 1$ and $q 2$, since we consider the same $\boldsymbol{Q}$ for both cases and, as explained in Section 4.1, the value of $\boldsymbol{K}$ is determined by $\boldsymbol{Q}$ and $R$ for the linear time invariant system. The initial errors for different spacecraft are chosen to be maximal in the controllable sense, this is, the trajectory escapes when we increase these errors.

From Table 5, it follows that the larger the nominal charge, the smaller the 

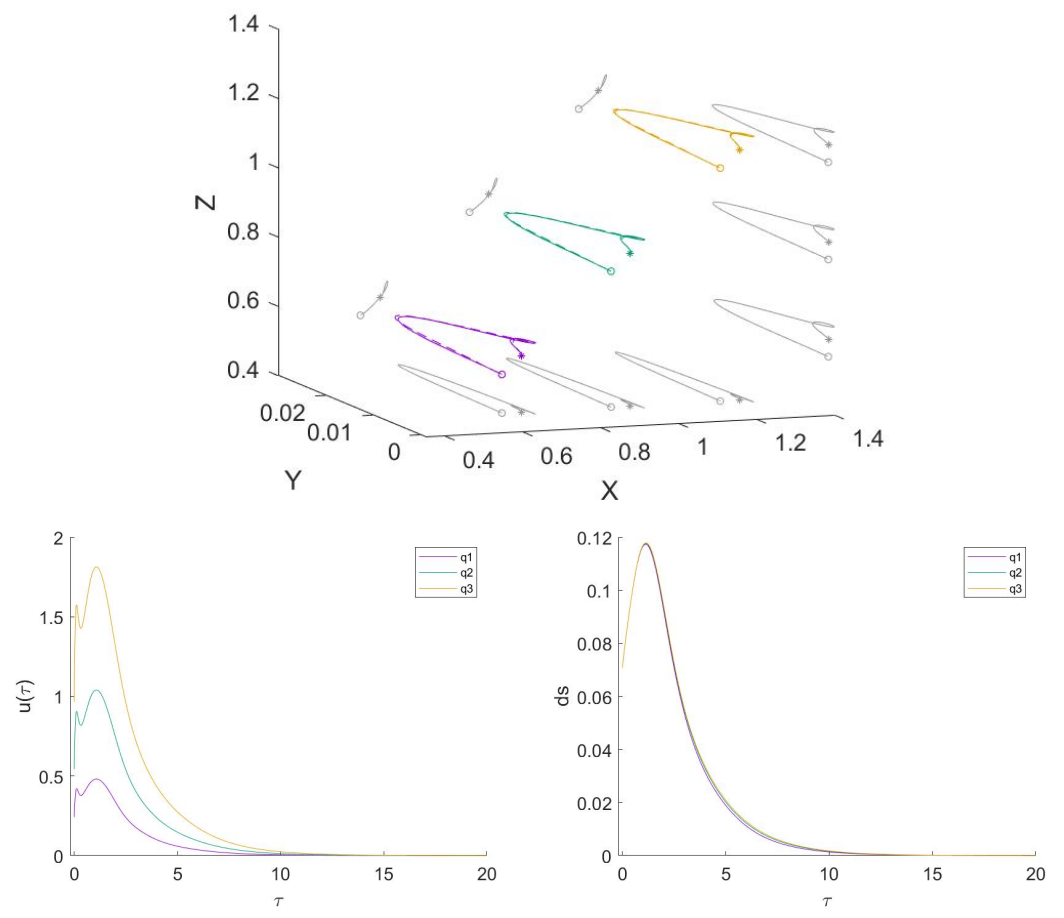

Figure 19: 3D view (coloured) and the three projections (grey) of the controlled trajectories of three spacecrafts in the linear (solid line) and non-linear (dashed line) systems (top row), time evolution of the control input $\eta \cdot u(\tau)$ (bottom left), and distance of the spacecraft to the target equilibrium point (bottom right) in the non-linear system. The colour code for the charge is $q 1$ in purple, $q 2$ and $q 3$ in green and brown, respectively. The computations are done with $\beta=2$, and departing from the equilibrium point $3_{R}^{1}$ (star) with a displacement equal to $(1,0,1,1,0,1)^{\top} \times 0.05$ (circle) .

Table 5: The values of initial error (modulus along the vector $(1,0,1,1,0,1)^{\top}$ ), weight $\boldsymbol{Q}$, and gain vector $\boldsymbol{K}$ for the three spacecrafts with different charges $\eta q^{\star}$.

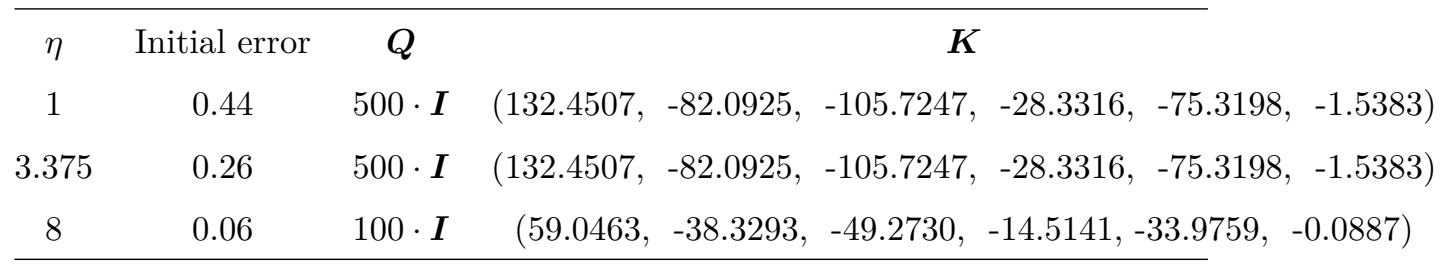

maximal initial error that can be controlled. The maximum controllable initial error for $\eta=3.375$ and $\eta=8$ are about $60 \%$ and $14 \%$ of the one associated 
to $\eta=1$. The difference in the maximal initial error is caused by the charge ratio $\eta$, as it is shown in Eq. (14). The ranges $\left[u_{\min }, u_{\max }\right]$ of the adimensional control charge for the three spacecraft are shown in Table 6, while the ones used for the computation of the control input and the integration of the controlled trajectories are in the second column. We see that the range of $u(\tau)$ for $\eta=1$ is $[-11,9]$, which is coherent with the ones given in Eq. (13), while the ones for $\eta=3.375$ and $\eta=8$ are only $30 \%$ and $12.5 \%$ of the ones associated to $\eta=1$. The controllability has a monotonic (yet not linear) dependence on the range $\left[u_{\min }, u_{\max }\right]$, which is determined by the value of $\eta$. We remark that if the bound restriction on the total charge is removed, and the control input is applied by means of Eq. (9), the maximal controllable initial errors could be the same for different values of $\eta$, however, this is not realistic from the practical technical point of view.

Table 6: Ranges of the adimensional control input for the three spacecraft with different charges; both in the units of $q_{i}$ (for integration) and of $q^{\star}$ (for comparison).

\begin{tabular}{ccc}
\hline$\eta$ & {$\left[u_{\min }, u_{\max }\right]\left(\right.$ unit: $\left.q_{i}\right)$} & {$\left[u_{\min }, u_{\max }\right]\left(\right.$ unit: $\left.q^{\star}\right)$} \\
\hline 1 & {$[-11,9]$} & {$[-11,9]$} \\
3.375 & {$[-3.963,1.963]$} & {$[-13.375,6.625]$} \\
8 & {$[-2.25,0.25]$} & {$[-18,2]$} \\
\hline
\end{tabular}

Figure 20 displays the controlled trajectories of the three considered spacecraft, together with the time evolution of the distance between either two spacecraft, the control charge $\eta \cdot u(\tau)$, and the displacements of each spacecrafts relative to the leader at the origin. It can be clearly seen that the three trajectories of the non-linear systems overlap each other and may exist collision risk between the spacecrafts, while the ones of the linear systems are separated. Due to the large initial errors considered, the controlled non-linear trajectories can move very far from the linear one (maximum values being 1.06, 0.6 and 0.14 for the $q 1, q 2$ and $q 3$ charges respectively), and are almost 2.5 times the order of the initial errors (resp. 0.44, 0.26 and 0.06). The same phenomena appeared in 
Fig. 16 as explained in Section. 4.3. No larger initial errors can be endured for controllability, as can be seen in Fig. 20 (bottom left). The required charges reach the lower bound in the time interval $[0,3]$, when the spacecraft also reach their maximum deviation. We remark that the controllability, as well as the rate of converge of the system, mainly depend on the stability of the reference equilibrium point, rather than on the charges and initial errors. This can be seen at the second row of Fig. 20, where all the evolution curves for the control input and displacements display similar behaviour for spacecrafts with different charges.

From figure 20 we can also see that, although there seems to appear a collision between trajectories (top left), actually no collision happens, since the distances between any two spacecraft are larger than 0.04 length units during the whole controlled process of formation keeping. However, we remark that collision risk may increase when more spacecraft, with smaller charge difference, are deployed or when different initial errors are considered. 

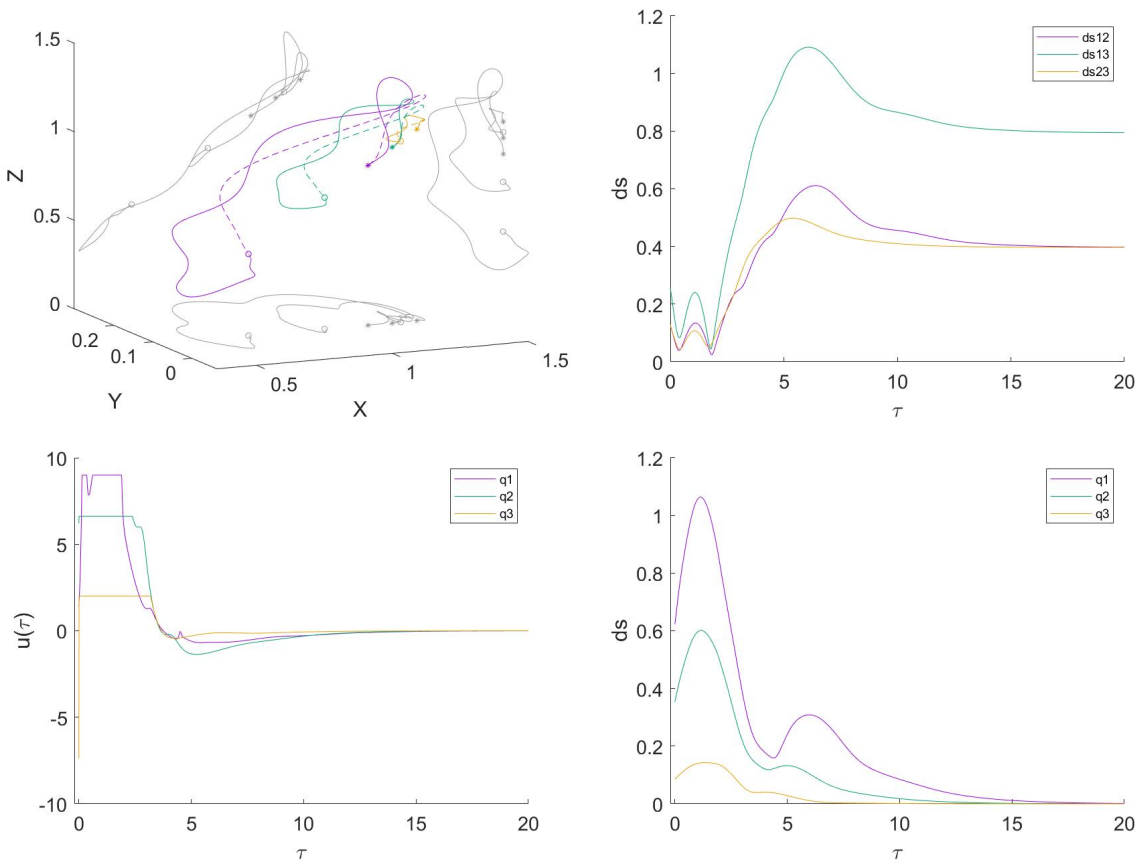

Figure 20: (Top left) 3D view and coordinate projections of the controlled trajectories of three spacecraft in the linear and non-linear systems. (Top right), time evolution of the distance between spacecraft pairs. (Bottom left), the control input $u(\tau)$. (Bottom right), distance of the spacecraft to the target equilibrium point. All them in the non-linear system defined by (3) and (5). The colour code for the trajectories is as follows: $q 1$ purple, $q 2$ green and $q 3$ brown. Computations are performed with $\beta=2$ departing from the equilibrium point $3_{R}^{1}$ (marked with a star) with the displacement specified in Table 5 (marked with a circle) .

\section{Discussion}

In this paper we have used adimensional units. According to Eq. (2), the physical distance unit is determined by the value of charge-to-mass ratio $\frac{q}{m}$, the magnetic field moment $B_{0}$, and the angular ratio $\beta$, while the time unit is independent of these parameters. The magnetic field strength $B_{0}$, as well as $\beta$, are considered fixed once the HTSC coil is designed, but $\frac{q}{m}$ remains adjustable.

The advantage of using adimensional units for the cases considered becomes clear since, with each value of $\beta$, we just need to compute one time the location of equilibria and the size of periodic orbits. Then, a simple scaling by the real 
distance unit provides orbits of different sizes. Therefore, once a simple configuration is designed in adimensional units, more configurations can be easily considered, just by means of adding spacecrafts with different charges, as shown in Fig. 3. Other options consist in the combinations of several simple configurations of any number and size. The flexibility of the proposed configurations provides a vast potential in multi-follower formation flying of the model studied by the authors in $[3,4]$.

However, collision risk arises when we have many follower spacecraft. We notice that when the spacecraft are of the same charge in the multi-follower configurations, the risk of collision is very small and can be neglected. In this case each spacecraft is located at different equilibrium point, the distance between any two equilibria are large, while each spacecraft move in a close region around its reference equilibrium point, even when an initial displacement is added. However, when the reference equilibrium point is the same and the charges of the deputies are different, the possibility of collision arises when the initial error is large. In this case, even if the spacecraft are independently controllable, the trajectory may depart far away from the reference equilibrium point as seen in Fig. 16. The collision avoidance requires more complicated control strategies than the one presented in the present paper; relative distances need to be monitored and more path constraints have to be considered. The control strategy including collision avoidance, as the one developed in [23], will be the focus of our future work.

\section{Conclusions}

In this paper we have explored the application to formation flying in a dynamical reference model that was thoroughly studied in [4]. Several charged follower spacecraft move around a leader in a high Earth orbit (such as GEO) that is provided with an artificial magnetic field, so the followers are subjected to the gravitational force from the Earth and to the Lorentz force produced by the leader's magnetic dipole. 
The proposed formation flying configurations consider the equilibrium points of the model as nominal locations for the deputies. In the ideal case, where no perturbations are considered, those kinds of configurations can be achieved with merely the assumption that the nominal charge $q^{\star}$ of the follower is electrostatically constant. Taking into account possible tracking or injection errors, the paper aims to examine the capacity of the self-maintenance of the formation configurations in the reference model, that is the Lorentz force is the only source of propulsion, which means that the charge of the follower will be the unique control parameter.

For this purpose, we have studied and analysed the evolution of the configurations in presence of initial errors, such as tracking errors or initial injection errors. If the equilibrium point has hyperbolic part, this is, at least one associated eigenvalue has non-zero real part, the spacecraft will escape soon after departing from this reference point, even if the initial error is as small as $1 \times 10^{-3}$; while for the totally elliptic equilibrium points, the configuration could persist for a long time with the same initial error (with each spacecraft moving on an invariant tori bounded in a region of the size in the same order of the initial displacement). However, a control strategy has to be included to avoid escaping if the initial error is increased, and also for the case when the equilibrium point has hyperbolic component.

The controllability of the linearised system around the nine kinds of equilibria has been explored. It has been shown that all but one kind $\left(3_{N}\right)$ of the equilibria are controllable. Using the charge of the spacecraft as the unique control input, a LQR feedback control law has been developed for the maintenance of configurations, including either single or multiple spacecraft. By means of varying the value of the initial error and the angular ratio $\beta$ between the mean motion of the leader's orbit and the rotation rate of the magnetic dipole, the controllability of the system has been examined. Different kinds of configurations, including one-follower and multiple deputies with the same charge or different charges, are considered. By means of tuning the value of $\boldsymbol{Q}$ in the feedback control law, the performance of the controllability has been optimized 
for the several configurations studied.

Numerical simulations show that the controllability is strongly related to the stability of the reference equilibrium point, which in turn depends on the value of $\beta$. When the reference equilibrium point is hyperbolic, the stability of the linearised and non-linear systems around this point are topologically the same and the configuration maintenance can be achieved using the linear feedback control. In this case, when the real part of the eigenvalues have very small amplitude, the controllability is very robust handling initial displacements as large as the order of 0.44 . No larger initial errors can be cancelled using only the charge control since, taking into account the current technical limitations on the spacecraft surface charge, we have set the bound of the total charge to be $-[10,10] * q^{\star}$.

Moreover, from a practical point of view, the totally elliptic equilibrium point (this is, when it has three pairs of purely imaginary eigenvalues), are not favourable if we want better controllability, since the trajectories dwindle around the reference equilibrium point for very long times. It is noted that the collision risk arises when the number of deputies, or the initial errors, increase; specially in the case where only one kind of equilibrium points is chosen as nominal location and multiple spacecrafts with different charges are deployed. However, one may avoid collisions by carefully choosing the charges to get nominal locations that are separated relatively far from each other.

We can conclude that, both the establishment and maintenance of the formation flying configurations can be implemented by merely adjusting the charge on the follower spacecraft; no additional propulsions are needed, which makes the Lorentz force coming from an artificial magnetic field a novel and promising way for future formation flying missions.

\section{Acknowledgements}

Y.C. and D.Q. thank the Discipline Innovative Engineering Plan (111 Project). G.G. thanks the Catalan grant government for the grant 2017SGR-1374 and 
MINECO-FEDER for the grant MTM2016-80117-P. J.J.M. thanks MINECOFEDER for the grant MTM2015-65715-P and the Catalan government for the grant 2017SGR-1049. Y.L. thanks the National Natural Science Foundation of China (grant 11602297).

\section{Appendix}

\section{The matrix $A$ and the vector $B_{q}$ of the linearised equations}

We denote the state vector as $\boldsymbol{X}=\left(X, Y, Z, X^{\prime}, Y^{\prime}, Z^{\prime}\right)^{\top}$, and treat the charge $q$ of the follower as control input that is time varying, and the equations of motion (3) can be rewritten in the form of the first-order differential equation, given by

$$
\boldsymbol{X}^{\prime}=\boldsymbol{F}(\boldsymbol{X}, q)
$$

and the components in the above equations are

$$
\begin{aligned}
& X^{\prime}=X^{\prime} \\
& Y^{\prime}=Y^{\prime} \\
& Z^{\prime}=Z^{\prime} \\
& X^{\prime \prime}=3 X-2 Y^{\prime}+f_{X}(\boldsymbol{X}, q) \\
& Y^{\prime \prime}=2 X^{\prime}+f_{Y}(\boldsymbol{X}, q) \\
& Z^{\prime \prime}=-Z+f_{Z}(\boldsymbol{X}, q)
\end{aligned}
$$

where $\left(f_{X}, f_{Y}, f_{Z}\right)$ are the adimensional components of the Lorentz force $\boldsymbol{f}_{L}$.

To study the controllability of the system around the equilibrium point, which is denoted as $X^{\star}$, we introduce the small perturbation $\Delta \boldsymbol{X}=\left(\boldsymbol{X}-\boldsymbol{X}^{*}\right)$ and the adimensional variation of the charge $\Delta q=\left(\frac{q}{q^{\star}}-1\right)$, and linearise Eq.(15) around the the equilibrium point $X^{\star}$ and the nominal charge $q^{\star}$,

$$
\Delta \boldsymbol{X}^{\prime}=\boldsymbol{A} \Delta X+\boldsymbol{B}_{q} \Delta q,
$$

which is the same as Eq.(6), and the coefficient matrix $\boldsymbol{A}=\left.\frac{d \boldsymbol{f}(\boldsymbol{X}, q)}{d \boldsymbol{X}}\right|_{\boldsymbol{X}^{\star}}$ is the derivative of the vector function $\boldsymbol{f}(\boldsymbol{X}, q)$ with respect to the state vector, 
evaluated at $\boldsymbol{X}^{\star}$, and vector coefficient $\boldsymbol{B}_{q}=\left.\frac{d \boldsymbol{f}(\boldsymbol{X}, q)}{d \boldsymbol{q}}\right|_{\boldsymbol{q}^{\star}}$ is the derivative of $\boldsymbol{f}(\boldsymbol{X}, q)$ with respect to the nominal charge $q^{\star}$. We note that if the charge $q$ of the follower is fixed as $q=q^{\star}$, the second term in the right-hand side of Eq.(17) will be zero, the follower will stay at the reference equilibrium point, and the linear equation will be the same as in [24].

Next, we will give the detailed expression of $\boldsymbol{A}$ and $\boldsymbol{B}_{q}$. From Eq. (16), it is obvious that the coefficient matrix $\boldsymbol{A}$ of the linear system (6) is of the form,

$$
\boldsymbol{A}=\left[\begin{array}{cccccc}
0 & 0 & 0 & 1 & 0 & 0 \\
0 & 0 & 0 & 0 & 1 & 0 \\
0 & 0 & 0 & 0 & 0 & 1 \\
a_{41} & a_{42} & a_{43} & a_{44} & a_{45} & a_{46} \\
a_{51} & a_{52} & a_{53} & a_{54} & a_{55} & a_{56} \\
a_{61} & a_{62} & a_{63} & a_{64} & a_{65} & a_{66}
\end{array}\right]
$$

where the first three rows are constants independent on the orientation of the magnetic dipole and the last three rows can be written as,

$$
\boldsymbol{A}([4,5,6],:)=\frac{d \boldsymbol{f}_{L}}{d \boldsymbol{X}}+\left[\begin{array}{cccccc}
3 & 0 & 0 & 0 & 2 & 0 \\
0 & 0 & 0 & -2 & 0 & 0 \\
0 & 0 & -1 & 0 & 0 & 0
\end{array}\right]
$$

where $\frac{d \boldsymbol{f}_{L}}{d \boldsymbol{X}}$ is the derivative of the Lorentz force $\boldsymbol{f}_{L}=\left(f_{X}, f_{X}, f_{Z}\right)^{\top}$ with respect to the state vector $\boldsymbol{X}=\left(X, Y, Z, X^{\prime}, Y^{\prime}, Z^{\prime}\right)^{\top}$, this is,

$$
\frac{d \boldsymbol{f}_{L}}{d \boldsymbol{X}}=\left[\begin{array}{llllll}
\frac{\partial f_{X}}{\partial X} & \frac{\partial f_{X}}{\partial Y} & \frac{\partial f_{X}}{\partial Z} & \frac{\partial f_{X}}{\partial X^{\prime}} & \frac{\partial f_{X}}{\partial Y^{\prime}} & \frac{\partial f_{X}}{\partial Z^{\prime}} \\
\frac{\partial f_{Y}}{\partial X} & \frac{\partial f_{Y}}{\partial Y} & \frac{\partial f_{Y}}{\partial Z} & \frac{\partial f_{Y}}{\partial X^{\prime}} & \frac{\partial f_{Y}}{\partial Y^{\prime}} & \frac{\partial f_{Y}}{\partial Z^{\prime}} \\
\frac{\partial f_{Z}}{\partial X} & \frac{\partial f_{Z}}{\partial Y} & \frac{\partial f_{Z}}{\partial Z} & \frac{\partial f_{Z}}{\partial X^{\prime}} & \frac{\partial f_{Z}}{\partial Y^{\prime}} & \frac{\partial f_{Z}}{\partial Z^{\prime}}
\end{array}\right]
$$

We note that the Lorentz force in components $\left(f_{X}, f_{Y}, f_{Z}\right)^{\top}$ is adimensional, while the one with subscripts $\{x, y, z\}$ correspond to real units.

Since the three components of Lorentz force $\left(f_{x}, f_{y}, f_{z}\right)$ in Eq. (2) share a similar pattern for all the three cases (normal, radial and tangential) considered, 
they can be written in a general form,

$$
f_{x i}=\frac{q}{m} \frac{B_{0}}{r^{3}}\left[\begin{array}{c}
3(\boldsymbol{N} \cdot \hat{\boldsymbol{r}})\left(\dot{x}_{i} \hat{r}_{i+1}-\dot{x}_{i+1} \hat{r}_{i}\right)+\dot{x}_{i+1} N_{i}-\dot{x}_{i} N_{i+1} \\
-\omega_{c}\left(x_{i+2} N_{i+1}-x_{i+1} N_{i+2}\right)\left(3(\boldsymbol{N} \cdot \hat{\boldsymbol{r}}) \hat{r}_{i+1}-N_{i+1}\right) \\
+\omega_{c}\left(x_{i} N_{i+2}-x_{i+2} N_{i}\right)\left(3(\boldsymbol{N} \cdot \hat{\boldsymbol{r}}) \hat{r}_{i}-N_{i}\right)
\end{array}\right]
$$

where $\boldsymbol{N}$ is the unit vector along the dipole orientation, and $\hat{\boldsymbol{r}}=\left(\frac{x}{r}, \frac{y}{r}, \frac{z}{r}\right)^{\top}$ with $r=\sqrt{x^{2}+y^{2}+z^{2}}$ is the normalized position vector of the spacecraft. The position components denoted by the subscripts follow the rules given in Table 7 , which also hold for the velocity components $\dot{x}, \dot{y}, \dot{z}$. We remark that the three basic $X, Y$ and $Z$ axes are aligned with the $x, y$ and $z$ axes, respectively, thus the position and velocity vector associated to $\left\{X_{i}, X_{i+1}, X_{i+2}\right\}$ follow the same rule defined in the same table.

Table 7: Position components denoted by $x_{i}, x_{i+1}$ and $x_{i+2}$ for the different orientation cases of the magnetic dipole $\boldsymbol{N}$.

\begin{tabular}{|c|c|c|c|c|c|c|}
\hline & & $\mathbf{N}$ & & $x_{i}$ & $x_{i+1}$ & $x_{i+2}$ \\
\hline Normal case & {$[0$} & 0 & 1] & $z$ & $x$ & $y$ \\
\hline Radial case & {$[1$} & 0 & 0] & $x$ & $y$ & $z$ \\
\hline Tangential case & {$[0$} & 1 & $0]$ & $y$ & $z$ & $x$ \\
\hline
\end{tabular}

Using this nomenclature, the dipole's orientation can be expressed as $\left(N_{i}=\right.$ $\left.\pm 1, \quad N_{i+1}=0=N_{i+2}=0\right)$, and the general form of the Lorentz force acceleration can be written as,

$$
\begin{aligned}
f_{X_{i}} & =\frac{q}{m} \frac{B_{0}}{r^{5}} \cdot 3 x_{i}\left[x_{i+2} \dot{x}_{i+1}-x_{i+1} \dot{x}_{i+2} \pm\left(x_{i+1}^{2}+x_{i+2}^{2}\right)\right] \\
f_{X_{i+1}} & =\frac{q}{m} \frac{B_{0}}{r^{5}}\left[\begin{array}{c}
-\left(x_{i+1}^{2}+x_{i+2}^{2}-2 x_{i}^{2}\right) \dot{x}_{i+2}-3 x_{i+2} x_{i} \dot{x}_{i} \\
\pm \omega_{c} x_{i+1}\left(x_{i+1}^{2}+x_{i+2}^{2}-2 x_{i}^{2}\right)
\end{array}\right] \\
f_{X_{i+2}} & =\frac{q}{m} \frac{B_{0}}{r^{5}}\left[\begin{array}{c}
\left(x_{i+1}^{2}+x_{i+2}^{2}-2 x_{i}^{2}\right) \dot{x}_{i+1}+3 x_{i+1} x_{i} \dot{x}_{i} \\
\pm \omega_{c} x_{i+2}\left(x_{i+1}^{2}+x_{i+2}^{2}-2 x_{i}^{2}\right)
\end{array}\right]
\end{aligned}
$$

Then, rescaling by the set of units defined in Eq. (2), the Lorentz-force 
acceleration can be rewritten as,

$$
\begin{aligned}
& f_{X_{i}}=\frac{1}{R^{5}} \cdot 3 X_{i}\left[\beta X_{i+2} X_{i+1}^{\prime}-X_{i+1} X_{i+2}^{\prime} \pm\left(X_{i+1}^{2}+X_{i+2}^{2}\right)\right] \\
& f_{X_{i+1}}=\frac{1}{R^{5}}\left[\begin{array}{c}
-\beta\left(X_{i+1}^{2}+X_{i+2}^{2}-2 X_{i}^{2}\right) X_{i+2}^{\prime}-3 \beta X_{i+2} X_{i} X_{i}^{\prime} \\
\pm \omega_{c} X_{i+1}\left(X_{i+1}^{2}+X_{i+2}^{2}-2 X_{i}^{2}\right)
\end{array}\right] \\
& f_{X_{i+2}}=\frac{1}{R^{5}}\left[\begin{array}{c}
\left(X_{i+1}^{2}+X_{i+2}^{2}-2 X_{i}^{2}\right) X_{i+1}^{\prime}+3 X_{i+1} x_{i} X_{i}^{\prime} \\
\pm \omega_{c} X_{i+2}\left(X_{i+1}^{2}+X_{i+2}^{2}-2 X_{i}^{2}\right)
\end{array}\right]
\end{aligned}
$$

Finally, the partial derivatives of the Lorentz force $\boldsymbol{f}_{L}=\left(f_{X_{i}}, f_{X_{i+1}}, f_{X_{i+2}}\right)^{\top}$ with respect to the state vector $\boldsymbol{X}=\left(X_{i}, X_{i+1}, X_{i+2}, X_{i}^{\prime}, X_{i+1}^{\prime}, X_{i+2}^{\prime}\right)^{\top}$, both expressed in general form, are given by,

$$
\begin{aligned}
\frac{\partial f_{X_{i}}}{\partial X_{i}} & = \pm A_{1} \cdot \frac{3}{R^{5}}\left(1-\frac{5 X_{i}}{R^{2}}\right) \\
\frac{\partial f_{X_{i}}}{\partial X_{i+1}} & = \pm A_{1} \frac{3 X_{i}}{R^{5}}\left(-\beta X_{i+2}^{\prime}+2 X_{i+1}-A_{1} \frac{5 X_{i+1}}{R^{2}}\right) \\
\frac{\partial f_{X_{i}}}{\partial X_{i+2}} & = \pm A_{1} \frac{3 X_{i}}{R^{5}}\left(\beta X_{i+1}^{\prime}+2 X_{i+2}-A_{1} \frac{5 X_{i+2}}{R^{2}}\right) \\
\frac{\partial f_{X_{i}}}{\partial X_{i}^{\prime}} & =0 \\
\frac{\partial f_{X_{i}}}{\partial X_{i+1}^{\prime}} & = \pm \frac{1}{R^{5}}\left(3 \beta X_{i} X_{i+2}\right) \\
\frac{\partial f_{X_{i}}}{\partial X_{i+2}^{\prime}} & = \pm \frac{1}{R^{5}}\left(-3 \beta X_{i} X_{i+1}\right)
\end{aligned}
$$

where $A_{1}=\beta X_{i+2} X_{i+1}^{\prime}-\beta X_{i+1} X_{i+2}^{\prime}+\omega_{c}\left(X_{i+1}^{2}-X_{i+2}^{2}\right)$.

$$
\begin{aligned}
& \frac{\partial f_{X_{i+1}}}{\partial X_{i}}= \pm \frac{1}{R^{5}}\left(4 \beta X_{i} X_{i+2}^{\prime}-3 \beta X_{i+2} X_{i}^{\prime}-4 X_{i} X_{i+1}-A_{2} \cdot \frac{5 X_{i}}{R^{2}}\right) \\
& \frac{\partial f_{X_{i+1}}}{\partial X_{i+1}}= \pm \frac{1}{R^{5}}\left(-2 \beta X_{i+1} X_{i+2}^{\prime}+3 X_{i+1}^{2}+X_{i+2}^{2}-2 X_{i}^{2}-A_{2} \cdot \frac{5 X_{i+1}}{R^{2}}\right) \\
& \frac{\partial f_{X_{i+1}}}{\partial X_{i+2}}= \pm \frac{1}{R^{5}}\left(-2 \beta X_{i+2} X_{i+2}^{\prime}-3 \beta X_{i} X_{i}^{\prime}+2 X_{i+1} X_{i+2}-A_{2} \cdot \frac{5 X_{i+2}}{R^{2}}\right) \\
& \frac{\partial f_{X_{i+1}}}{\partial X_{i}^{\prime}}= \pm \frac{1}{R^{5}}\left(-3 \beta X_{i} X_{i+2}\right) \\
& \frac{\partial f_{X_{i+1}}}{\partial X_{i+1}^{\prime}}=0 \\
& \frac{\partial f_{X_{i+1}}}{\partial X_{i+2}^{\prime}}= \pm \frac{1}{R^{5}}\left[-\beta\left(X_{i+1}^{2}+X_{i+2}^{2}-2 X_{i}^{2}\right)\right]
\end{aligned}
$$


where $A_{2}=-\beta\left(X_{i+1}^{2}+X_{i+2}^{2}-2 X_{i}^{2}\right) X_{i+2}^{\prime}-3 \beta X_{i+2} X_{i} X_{i}^{\prime}+X_{i+1}\left(X_{i+1}^{2}+X_{i+2}^{2}-2 X_{i}^{2}\right)$.

$$
\begin{aligned}
& \frac{\partial f_{X_{i+2}}}{\partial X_{i}}= \pm \frac{1}{R^{5}}\left(-4 \beta X_{i} X_{i+1}^{\prime}+3 \beta X_{i+1} X_{i}^{\prime}-4 X_{i} X_{i+2}-A_{3} \cdot \frac{5 X_{i}}{R^{2}}\right) \\
& \frac{\partial f_{X_{i+2}}}{\partial X_{i+1}}= \pm \frac{1}{R^{5}}\left(2 \beta X_{i+1} X_{i+1}^{\prime}+3 \beta X_{i} X_{i}^{\prime}+2 X_{i+1} X_{i+2}-A_{3} \cdot \frac{5 X_{i+1}}{R^{2}}\right) \\
& \frac{\partial f_{X_{i+2}}}{\partial X_{i+1}}= \pm \frac{1}{R^{5}}\left(2 \beta X_{i+2} X_{i+1}^{\prime}+3 X_{i+2}^{2}+X_{i+1}^{2}-2 X_{i}^{2}-A_{3} \cdot \frac{5 X_{i+2}}{R^{2}}\right) \\
& \frac{\partial f_{X_{i+2}}}{\partial X_{i}^{\prime}}= \pm \frac{1}{R^{5}}\left(-3 \beta X_{i} X_{i+1}\right) \\
& \frac{\partial f_{X_{i+2}}}{\partial X_{i+1}^{\prime}}= \pm \frac{1}{R^{5}}\left[\beta\left(X_{i+1}^{2}+X_{i+2}^{2}-2 X_{i}^{2}\right)\right] \\
& \frac{\partial f_{X_{i+2}}}{\partial X_{i+2}^{\prime}}=0
\end{aligned}
$$

where $A_{3}=\beta\left(X_{i+1}^{2}+X_{i+2}^{2}-2 X_{i}^{2}\right) X_{i+1}^{\prime}-3 \beta X_{i+1} X_{i} X_{i}^{\prime}+X_{i+2}\left(X_{i+1}^{2}+X_{i+2}^{2}-2 X_{i}^{2}\right)$.

If we follow the sequence $X_{i} \rightarrow X_{i+1} \rightarrow X_{i+2}$ to compute the above partial derivatives, we get exactly the ones for the radial case. However, for the tangential case with $\boldsymbol{N}=(0,1,0)^{\top}$, the equations (20), (21) and (22) give us,

$$
\frac{d \boldsymbol{f}_{L T}}{d \boldsymbol{X}_{T}}=\left[\begin{array}{llllll}
\frac{\partial f_{Y}}{\partial Y} & \frac{\partial f_{Y}}{\partial Z} & \frac{\partial f_{Y}}{\partial X} & \frac{\partial f_{Y}}{\partial Y^{\prime}} & \frac{\partial f_{Y}}{\partial Z^{\prime}} & \frac{\partial f_{Y}}{\partial X^{\prime}} \\
\frac{\partial f_{Z}}{\partial Y} & \frac{\partial f_{Z}}{\partial Z} & \frac{\partial f_{Z}}{\partial X} & \frac{\partial f_{Z}}{\partial Y^{\prime}} & \frac{\partial f_{Z}}{\partial Z^{\prime}} & \frac{\partial f_{Z}}{\partial X^{\prime}} \\
\frac{\partial f_{X}}{\partial Y} & \frac{\partial f_{X}}{\partial Z} & \frac{\partial f_{X}}{\partial X} & \frac{\partial f_{X}}{\partial Y^{\prime}} & \frac{\partial f_{X}}{\partial Z^{\prime}} & \frac{\partial f_{X}}{\partial X^{\prime}}
\end{array}\right]
$$

where the subscript $T$ stands for the tangential case.

In order to get the real form of $\frac{d \boldsymbol{f}_{L}}{d \boldsymbol{X}}$, we need to perform a row switching transformation $\left(R_{1}, R_{2}, R_{3}\right) \rightarrow\left(R_{2}, R_{3}, R_{1}\right)$, followed by a column switching transformation $\left(C_{1}, C_{2}, C_{3}, C_{4}, C_{5}, C_{6}\right) \rightarrow\left(C_{2}, R_{3}, R_{1}, C_{5}, C_{6}, C_{4}\right)$, where $R_{i}, C_{i}(i \in\{1,2,3,4,5,6\})$ stands for the line and the row numbers, respectively.

For the normal case with $\boldsymbol{N}=(0,0,1)^{\top}$, using the equations (20), (21) 
and (22), we obtain,

$$
\frac{d \boldsymbol{f}_{L N}}{d \boldsymbol{X}_{N}}=\left[\begin{array}{llllll}
\frac{\partial f_{Z}}{\partial Z} & \frac{\partial f_{Z}}{\partial X} & \frac{\partial f_{Z}}{\partial Y} & \frac{\partial f_{Z}}{\partial Z^{\prime}} & \frac{\partial f_{Z}}{\partial X^{\prime}} & \frac{\partial f_{Z}}{\partial Y^{\prime}} \\
\frac{\partial f_{X}}{\partial Z} & \frac{\partial f_{X}}{\partial X} & \frac{\partial f_{X}}{\partial Y} & \frac{\partial f_{X}}{\partial Z^{\prime}} & \frac{\partial f_{X}}{\partial X^{\prime}} & \frac{\partial f_{X}}{\partial Y^{\prime}} \\
\frac{\partial f_{Y}}{\partial Z} & \frac{\partial f_{Y}}{\partial X} & \frac{\partial f_{Y}}{\partial Y} & \frac{\partial f_{Y}}{\partial Z^{\prime}} & \frac{\partial f_{Y}}{\partial X^{\prime}} & \frac{\partial f_{Y}}{\partial Y^{\prime}}
\end{array}\right]
$$

where the subscript $N$ stands for the normal case.

Two transformation steps are required to obtain the real form of $\frac{d \boldsymbol{f}_{L}}{d \boldsymbol{X}}$. The first one is a row switching transformation $\left(R_{1}, R_{2}, R_{3}\right) \rightarrow\left(R_{3}, R_{1}, R_{2}\right)$, while the second one is a column switching transformation $\left(C_{1}, C_{2}, C_{3}, C_{4}, C_{5}, C_{6}\right) \rightarrow$ $\left(C_{3}, R_{1}, R_{2}, C_{6}, C_{4}, C_{5}\right)$.

Then, the matrix $\boldsymbol{A}$ can be obtained by substituting the partial derivative $\frac{d f_{L}}{d X}$ into Eq. (18).

To compute the coefficient vector $\boldsymbol{B}_{q}$, we first rewrite the Lorentz force as a function of the charge $q$ and the nominal charge $q^{\star}$, given by

$$
\begin{aligned}
f_{X} & =\frac{\sigma}{R^{5}} g_{x}^{\star} \cdot \frac{q}{q^{\star}} \\
f_{Y} & =\frac{\sigma}{R^{5}} g_{y}^{\star} \cdot \frac{q}{q^{\star}} \\
f_{Z} & =\frac{\sigma}{R^{5}} g_{z}^{\star} \cdot \frac{q}{q^{\star}}
\end{aligned}
$$

where $R=\sqrt{X^{2}+Y^{2}+Z^{2}}$ is the distance between the follower and the leader, and $\sigma$ is the sign of the charge of the follower, and $\left(g_{x}^{\star}, g_{y}^{\star}, g_{z}^{\star}\right)$ are given in Eqs.(5). We note that if the charge of the follower is kept fixed as the nominal one such that $q=q^{\star}$, the follower will stay at the reference equilibrium point.

Using the same nomenclature as in the computation of matrix $\boldsymbol{A}$, we can obtain the coefficient vector $\boldsymbol{B}_{q}$ from Eq.(16) and (23), which can be expressed in a general form,

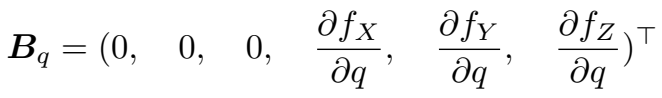

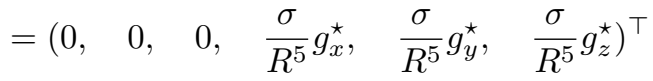

where the adimensional nominal charge $q^{\star}=1$, and the last three components 
are the Lorentz force given in Eq.(4) that corresponds to $q=q^{\star}$, the definition of each parameter in the above equation (24) is referred therein.

\section{Reference}

[1] J. Atchison, M. Peck. Lorentz-augmented Jovian orbit insertion. Journal of Guidance, Control, and Dynamics, 32(2):418-425, 2009.

[2] B. Baudouy. Heat transfer and cooling techniques at low temperature. arXiv preprint arXiv:1501.07153, 2015.

[3] Y. Cheng, G. Gómez, J. J. Masdemont, J. Yuan. Formation Flying in Space-Borne Artificial Magnetic Dipole Field. Joint Conference 31st ISTS, 26th ISSFD \& 8th NSAT, Matsuyama, Japan, June 2017.

[4] Y. Cheng, G. Gómez, J. J. Masdemont and J. Yuan. Analysis of the relative dynamics of a charged spacecraft moving under the influence of a magnetic field. Communications in Nonlinear Science and Numerical Simulation,62:307-338, 2018.

[5] J. W.Gangestad, G. E. Pollock, J. M. Longuski. Propellantless stationkeeping at Enceladus via the electromagnetic Lorentz force. Journal of Guidance, Control, and Dynamics, 32(5):1457-1466, 2009.

[6] J. Gangestad, G. E. Pollock, J. Longuski. Lagrange's Planetary Equations for the Motion of Electrostatically Charged Spacecraft, Celestial Mechanics and Dynamical Astronomy,108(2)::125-145, 2010.

[7] J. W. Gangestad, G.E. Pollock, J. M. Longuski. Analytical expressions that characterize propellantless capture with electrostatically charged spacecraft. Journal of Guidance, Control, and Dynamics, 34(1):247-258, 2011.

[8] M. E. Hough. Lorentz force perturbations of a charged ballistic missile. Proceedings of the AIAA Guidance and Control Conference, AIAA-19821549, San Diego, CA, 1982. 
[9] J. K. Hunter, Introduction to dynamical systems, UC Davis Mathematics MAT 207A, 2011. Available online at https://www.math.ucdavis.edu/ hunter/m207/m207.pdf

[10] J. D. Jackson. Classical Electrodynamics, 3rd Edition, John Wiley \& Sons, Inc, 1999.

[11] D. W. Kwon. Propellantless formation flight applications using electromagnetic satellite formations. Acta Astronautica, 67(9-10):1189-1201, 2010.

[12] E. Kong, D. Kwon, S. Schweighart, et al. Electromagnetic formation flight for multi-satellite arrays. Journal of Spacecraft and Rockets, 41(4):659-666, 2004.

[13] D. W. Kwon. Propellantless formation flight applications using electromagnetic satellite formations. Acta Astronautica, 67(9-10):1189-1201, 2010.

[14] D. W. Kwon, R. J. Sedwick. Cryogenic heat pipe for cooling hightemperature superconducting coils. Journal of Thermophysics and Heat Transfer, 23(4):732-740, 2012.

[15] M. Bengtson, K. Wilson, J. Hughes, et al. Survey of the electrostatic tractor research for reorbiting passive GEO space objects. Astrodynamics, 2(4):291-305, 2018.

[16] M. A. Peck. Prospects and challenges for Lorentz-augmented orbits. Proceedings of the AIAA Guidance, Navigation, and Control Conference, AIAA-2005-5995, San Francisco, CA, 2005.

[17] M. A. Peck, B. Streetman, C. M. Saaj, et al. Spacecraft formation flying using Lorentz forces. Journal of British Interplanetary Society, 60(7):263$267,2007$.

[18] Wade Pulliam. Catcher's Mitt Final Report, DARPA, 2011. 
[19] A. K. Porter, D. J. Alinger, R. J. Sedwick, et al. Demonstration of electromagnetic formation flight and wireless power transfer. Journal of Spacecraft and Rockets, 51(6):1914-1923, 2014.

[20] G.E. Pollock, J.W. Gangestad, J.M. Longuski. Inclination change in lowEarth orbit via the geomagnetic Lorentz force. Journal of Guidance, Control, and Dynamics, 33(5):1387-1395, 2010.

[21] G. E. Pollock, J. Gangestad, J. Longuski. Analytical Solutions for the Relative Motion of Spacecraft Subject to Lorentz-Force Perturbations. Acta Astronautica, 68(1):204-217, 2011.

[22] C. Peng, Y. Gao. Lorentz-force-perturbed orbits with application to J2invariant formation. Acta Astronautica, 77:12-28, 2012.

[23] M. Li, H. Peng, W. Zhong. Optimal control of loose spacecraft formations near libration points with collision avoidance. Nonlinear Dynamics, 83(4):2241-2261, 2016.

[24] C. Peng. Relative orbital motion of a charged object near a spaceborne radially-directed rotating magnetic dipole. 66th International Astronautical Congress, Jerusalem, 2015.

[25] C. Peng, Y. Gao. Formation-flying planar periodic orbits in the presence of inter-satellite Lorentz force. IEEE Transactions on Aerospace and Electronic Systems, 53(3) 1412-1430 2017.

[26] L. Schaffer, J. A. Burns. Charged dust in planetary magnetospheres: Hamiltonian dynamics and numerical simulations for highly charged grains. Journal of Geophysical Research, 9(A9):17211-17223, 1994.

[27] C. M. Saaj, V. Lappas, D. Richie, M. Peck, B. Streetman, and H. Schaub. Electrostatic forces for satellite swarm navigation and reconfiguration Final report. Final Report for Ariadna Study Id. AO491905, 2006. 
[28] L. A. Sobiesiak, C. J. Damaren. Controllability of Lorentz-augmented spacecraft formations. Journal of Guidance Control and Dynamics, 38(11):2188-2195, 2015.

[29] B. Streetman, M. A. Peck. New synchronous orbits using the geomagnetic Lorentz force. Journal of Guidance, Control and Dynamics, 30(6):16771690, 2007.

[30] B. Streetman, M. A. Peck. A General Bang-Bang Control Method for Lorentz Augmented Orbits. AAS Spaceflight Mechanics Meeting, AAS Paper 08-111, Galveston, Texas, 2008

[31] B. Streetman, M. A. Peck. Gravity-assist maneuvers augmented by the Lorentz force. Journal of Guidance, Control, and Dynamics, 32(5):16391647, 2009.

[32] L. A. Sobiesiak, C. J. Damaren. Controllability of Lorentz-augmented spacecraft formations. Journal of Guidance Control and Dynamics, 38(11):2188-2195, 2015.

[33] L. A. Sobiesiak, C.J. Damaren. Lorentz-Augmented Spacecraft Formation Reconfiguration. IEEE Transactions on Control Systems and Technology, 24(2):514-524, 2016.

[34] S. Tsujii, M. Bando, H. Yamakawa. Spacecraft formation flying dynamics and control using the geomagnetic Lorentz force. Journal of Guidance, Control, and Dynamics, 36(1):136-148, 2013.

[35] S. P. Bhat. Controllability of nonlinear time-varying systems: Application to spacecraft attitude control using magnetic actuation. IEEE Transactions on Automatic Control, 50(11):1725-1735, 2005.

[36] Y. Yang. Controllability of spacecraft using only magnetic torques. IEEE Transactions on Aerospace and Electronic Systems, 52:954-961, 2016. 
[37] Y. Yang. An efficient algorithm for periodic Riccati equation with periodically time-varying input matrix. Automatica, 78:103-109, 2017.

[38] A. Umair, D. W. Miller, J. L. Ramirez. Control of electromagnetic satellite formations in near-Earth orbit. Journal of Guidance, Control and Dynamics, 33(6):1883-1891, 2010.

[39] Y. Yan, X. Huang, Y. Yang. Dynamics and Control of Lorentz-Augmented Spacecraft Relative Motion. Springer, 2017. 